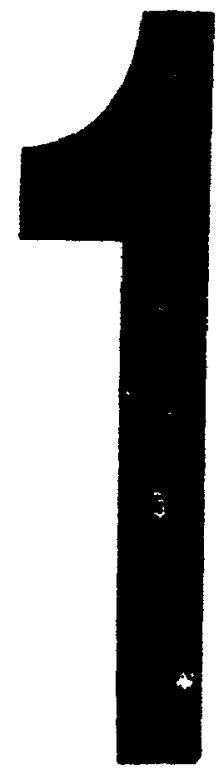

PM-1 3\%2" $x 4$ " PHOTOGRAPHIC MICROCOPY TARGET NBS 1010a ANS1/ISO \#2 EQUIVALENT

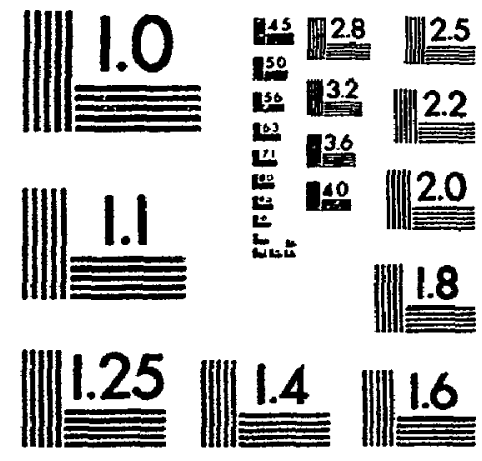


National Library

of Canada

Canadian Theses Service

Ottawa, Canada

K1A ON4
Bibliothèque nationale du Canada

\section{Service des theses canadiennes}

\section{NOTICE}

The quality of this microform is heavily dependent upon the quality of the original thesis submitted for microfilming. Every effort has been made to ensure the highest quality of reproduction possible.

If pages are missing, contact the university which granted the degree.

Some pages may have indistinct print especially if the original pages were typed with a poor typewriter ribbon or if the university sent us an inferior photocopy.

Reproduction in full or in part of this microform is governed by the Canadian Copyright ACt, R.S.C. 1970, c. C-30, and subsequent amendments.

\section{AVIS}

La qualité de cette microlorme dépend grandement de la qualite de la thèse soumise au microfilmage. Nous avons tout fait pour assurer une qualité supérieure de reproduction.

S'il manque des pages, veuillez communiquer avec luniversité qui a conféré le grade.

La qualité d'impression de certaines pages peut laisser à désirer, surtout si les pages originales ont été dactylographiées à l'aide d'un ruban usé ou si luniversité nous a lait parvenir une photocopie de qualité inférieure.

La reproduction, méme partielle, de cette microforme est soumise à la Loi canadienne sur le droit d'auteur, SRC 1970, c. C.30, et ses amendements subséqu'ents. 


\title{
THE ELECTROPHYSIOLOGICAL CORRELATES OF STAGE 2 DREAM RECALL
}

b y

Christine Morel

\author{
A thesis submitted to \\ the Faculty of Graduate Studies and Research \\ in partial fulfilment of \\ the requirements for the degree of \\ Doctor of Philosophy
}

\author{
Department of Psychology \\ Carleton University \\ Ottawa, Ontario \\ July 9,1991 \\ (C) Copyright
}


Canadian Theses Service Service des thèses canadiennes

Ottawa. Canada

KIA ON4

The author has granted an irrevocable nonexclusive licence allowing the National Library of Canada to reproduce, loan, distribute or sell copies of his/her thesis by any means and in any form or format, making this thesis available to inte:ested persons.

The author retains ownership of the copyright in his/her thesis. Neither the thesis nor substantial extracts from it may be printed or otherwise reproduced without his/her per. mission.
L'auteur a accordé une licence irrévocable et non exclusive permettant à la Bibliothèque nationale du Cansda de reproduire, preter. listribuer ou vendre des copies de sa these de quelque manière et sous quelque forme que ce soit pour mettre des exemplaires de cette thèse à la disposition des personnes intéressées.

L'auteur conserve la propriété du droit d'auteur qui protége sa thèse. Ni la thèse ni des extraits substantiels de celle-ci ne doivent être imprimés ou autrement reproduits sans son autorisation. 
The undersigned recommend to the Faculty of Graduate Studies and Research acceptance of the thesis submitted by

Christine R. More1

in partial fulfilment of the requirements for

the degree of

Doctor of PhIlosophy

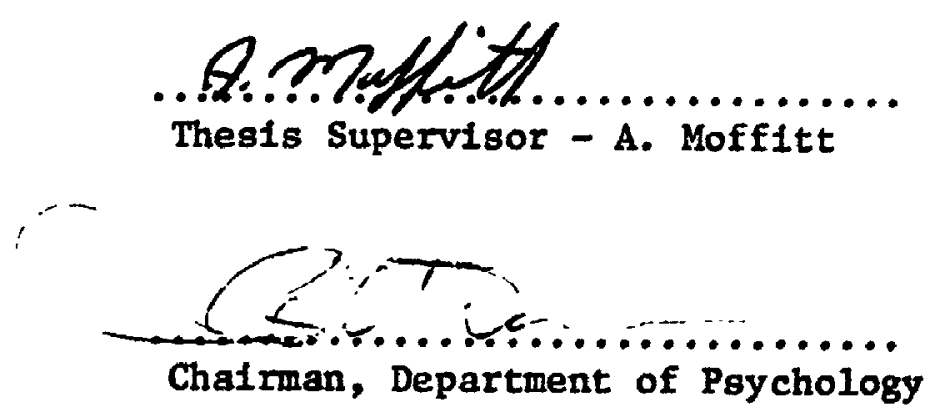


Recent proposals by Koukkou and Lehmann (1983; in press) have suggested a state-dependent relationship between cortical activation, defined by electroencephalographic (EEG) measures, and dream recall. This study investigated whether varying levels of cortical activity indexed by period-analyzed EEG measures were associated with dream recall performance for subjects awakened from stage 2 sleep.

Forty right-handed subjects (20 males, 20 females) spent one night each in the Sleep laboratory. The EEG data recorded from parietal electrode placements were examined for the 5 minute preawakening and 30 second postawakening time intervals. In addition, preawakening to postawakening EEG difference scores were computed. Principally, this research evaluated EEG differences between successful recall and failure to recall. The intermediate conditions of delayed success and contentless recall were also examined. In addition, the effects of gender in relation to dream recall (success vs failure) were assessed.

Findings indicated successful recall differed from failure to recall at the postawakening and preawakening to postawakening periods on measures of muscle activity and time spent in the sigma frequency band $(12 \mathrm{~Hz}<16 \mathrm{~Hz})$. There were significant differences between contentless and failure to recall groups at tho preawakening interval on the measure of muscle activity, as well as at the postawakening interval on the theta zero cross asymmetry measure. 
Finally, results revealed significant gender by recall interactions at the preawakening and postawakening periods.

Generally, the findings did not support the hypothesis linking increased dream recall to increases in cortical activation prior to awakening. Rather, the relatively similar EEG state during the preawakening dream production and encoding period would suggest that dreaming may be equally ongoing for all categories of recall. The lack of differences between recall groups on any of the preawakening and postawakening laterality measures, with the possible exception of contentless reporting, would suggest no strong differential involvement of the hemispheres in relation to dream recall. Overall, the main findings differentiating successful recall from failure to recall at the postawakening interval implies that dream recall performance may primarily be a postsleep process. Therefore, future research may benefit from the examination of changes in the pattern of arousal from the dreaming to the waking state. 


\section{ACKNOWLEDGEMENTS}

I would like to thank the members of my examination committee: Dean J.W. ApSimon, Dr. J. Campbell, Dr. R. Pigeau, Dr. H. Burshtyn, Dr. W. Walther, Dr. R. Hoffmann and Dr. A. Moffitt, for making the defense a most enjoyable experience. I wish to thank my advisor Dr. Alan Moffitt, for his astute comments, suggestions and continuing encouragement throughout my studies. I also wish to thank my co-advisor Dr. Robert Hoffmann, for his expertise at translating ideas into EEG-quantified data, for his support over the years and assistance in completing this paper. Many thanks to Dr. Ross Pigeau for ably handling the combined roles of former colleague and external examiner. I wish to extend my gratitude to Penny McGregor for rating the dream reports with diligence. Thanks to the members of the Sleep Lab, John Laing, Judy Niles and Frank Wimmer for their assistance and company during the long nights of recording. Special thanks to Rick Bonato, always willing to help: I appreciated your companionship and cheery disposition. Endless thanks to Charles Jones for his kindness and patience, for the long hours spent discussing, reviewing and editing this and all other texts, and for remaining with me through it all. My warmest thanks to Renée Sylvester for the enduring friendship and trust that I was and am still so fortunate to share. To my family, thanks for their support and care, and to my mother, special thanks for her concern and kind words. 


\section{TABLE OF CONTENTS}

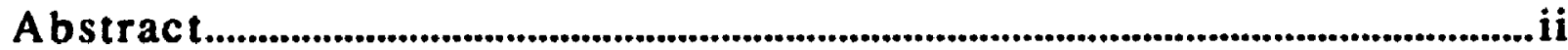

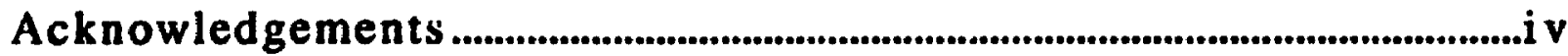

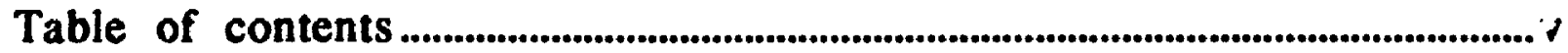

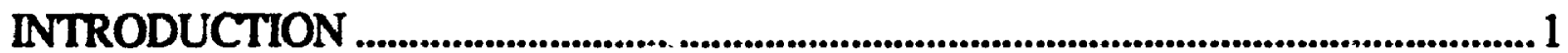

\section{CHAPTER ONE}

1.1. Sleep stage EEG and dream recall: Theory and research............5

1.2. Computer-quantified EEG variables and dream recall: Theoretical models ................................................................................................... 14

1.3. Computer-quantified EEG variables and dream recall: Empirical research...................................................................................................... 24

1.4. Hemispheric EEG variables and dream recall: Theoretical issues.......................................................................................... 32

1.5. Hemispheric EEG variables and dream recall:

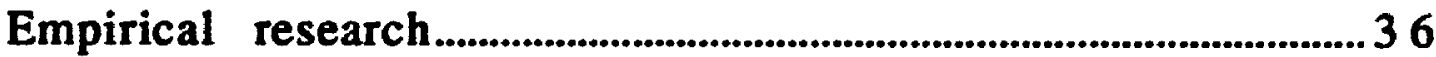

1.6. Dream recall and EEG: Further considerations ..............................4 41

\section{CHAPTER TWO}

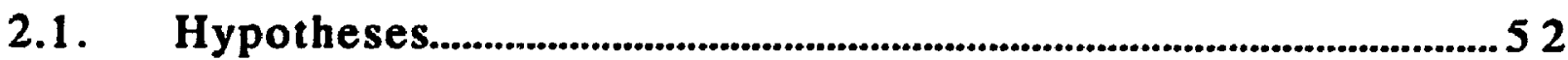

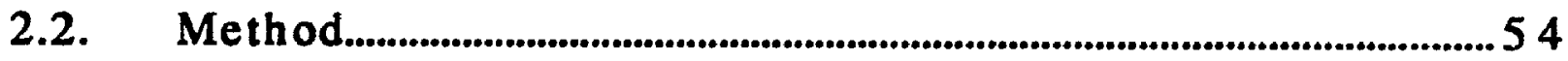

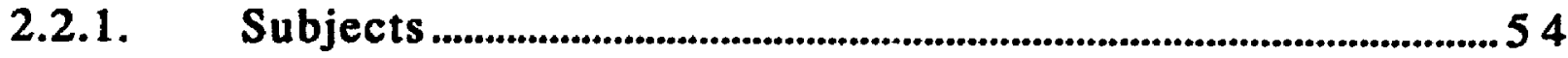

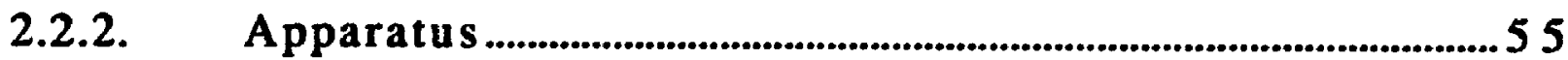

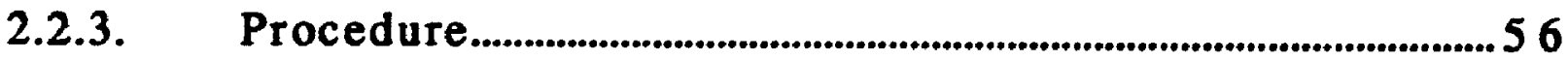

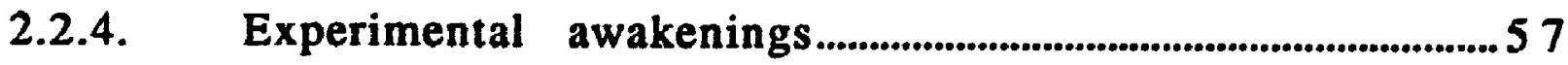

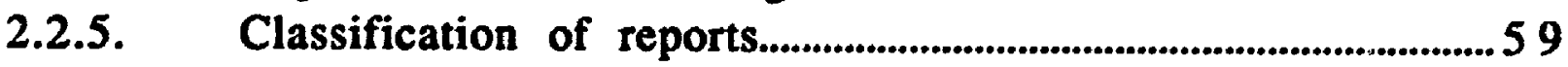

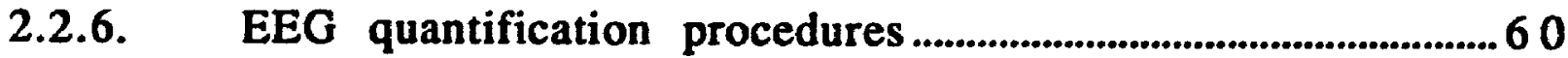

2.2.7. Statistical analyses (clear success vs failure)........................63

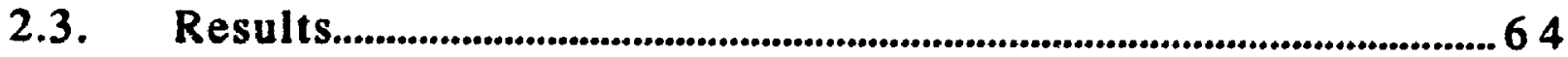

2.3.1. Dream recall: Descriptives ................................................................64 64

2.3.2. Dream recall and wakeup ordinal position.................................65 65

2.3.3. Gender and dream recall: Descriptives ........................................ 68 
2.3.4. Preawakening EEG and dream recall:

Clear success vs failure.

2.3.5. Postawakening EEG and dream recall:

Clear success vs failure

2.3.6. Preawakening to postawakening EEG differences and dream recall: Clear success vs failure.................................6 69

2.3.7. Within subject analysis: Clear success vs failure................... 7 I

2.3.8. Gender and dream recall ........................................................... 71

2.3.9. Multivariate analyses: Clear success vs failure.....................75

2.3.10. Other analyses............................................................................ 77

2.4. Discussion and implications of findings....................................... 79

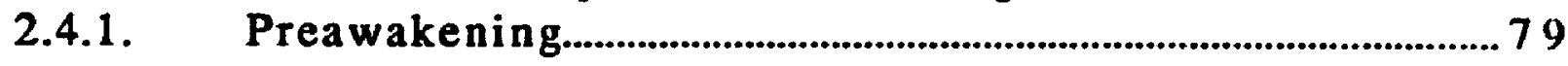

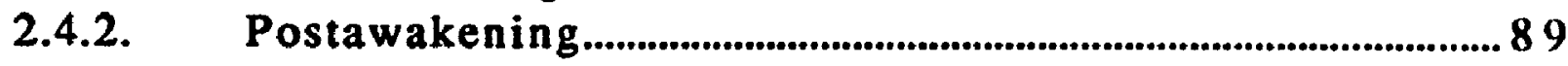

2.4.3. Preawakening to postawakening difference scores............. 94

2.4.4. Contentless and delayed success recall groups.......................95

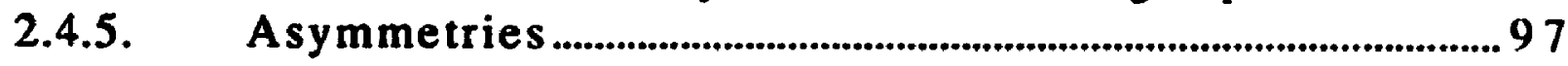

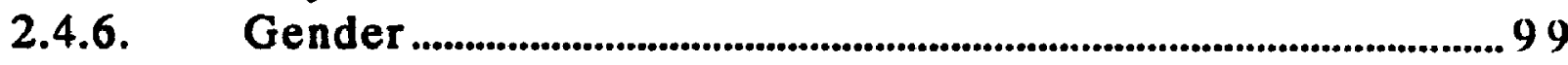

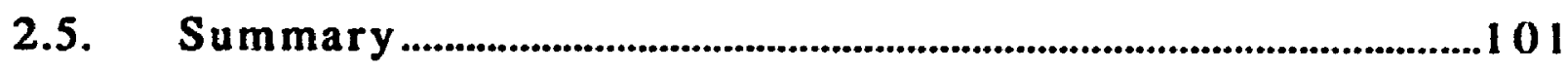

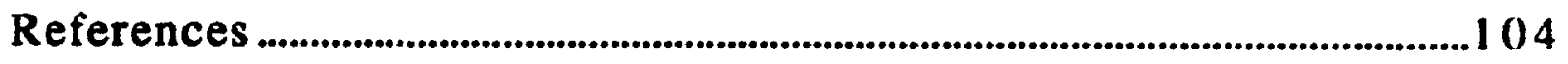

Appendix A Research application form ............................................119

Appendix B Follow-up information......................................................120

Appendix C Instructions upon arrival at the Lab...........................121

Appendix D Consent form.................................................................................122

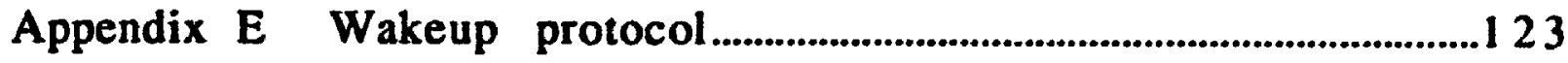

Appendix F The self-consciousness scale ............................................12 124

Appendix $G$ Instructions to raters.....................................................126 
INTRODUCTION

The purpose of this paper was to examine the relationship between cortical activation as indexed by electroencephalographic (EEG) measures and dream recall performance. This study investigated whether varying levels of cortical activity, indexed by period-analyzed EEG measures were associated with dream recall for subjects awakened from stage 2 sleep. The strategy of this research was to evaluate EEG differences between successful recall (reports with some content immediately upon awakening) and failure to recall (inability to recall anything). These two conditions theoretically represent the extremes of recall performance and are thus the most likely to reveal differences in cortical activation. The intermediate categories of delayed success and contentless recall were also examined. The correlates of recall performance were assessed using the preawakening, postawakening and preawakening to postawakening difference score sleep EEG measures to index the level of cortical activation.

The general research question addressed by this study was as follows: within a given stage of sleep, were characteristic patterns of EEG-defined cortical activation associated with corresponding changes in dream recall performance? The functional state-shift hypothesis of Koukkou and Lehmann (1980; 1983) provided the theoretical background in terms of which the proposed association between EEG-defined states and dream recall was evaluated. The assertions provided by their model are EEG-based and thus amenable 
to verification using computer analyses to characterize quantitative changes in EEG activity.

The electroencephalogram consists of recorded patterns of brain electrical activity and as such is thought to reflect the general arousal or activation level of the cerebral cortex. The fundamental assumption of models based on cortical activation is that during sleep, increasing levels of cortical activity as measured by the EEG. are associated with increasing levels of information processing in the form of dreaming and dream recall. However, attempts to explain dream recall performance in relation to EEG-defined cortical activation have generally failed to provide evidence of any consistent association.

One of the major limiting factors in defining the relationship between cortical arousal and recall performance was the measurement technique used to characterize the EEG patterns. In earlier studies, the use of a visual stage scoring procedure restricted the evaluation of changes in arousal levels to distinctions between sleep stages. By definition, visual stage scoring does not allow the evaluation of EEG fluctuations within a given stage. The use of computer techniques in sleep research has allowed a more detailed examination of EEG characteristics than was permitted using the visual stage scoring method (Moffitt \& Hoffmann, 1987). However, of the few dream recall studies available, the majority applied spectral analysis to the EEG waveforms, a method yielding only EEG power measures.

Another limitation in relating recall to EEG measures is the 
paucity of research examining dream recall out of non-REM sleep (non-rapid eye movement). Unlike REM, non-REM sleep, stage 2 specifically, occurs regularly across the night, and may provide a better distribution of recall success and failure.

Finally, following various theoretical assumptions (Antrobus, 1986; Koukkou \& Lehmann, 1983) research linking cortical activation to dream recall has restricted analyses to the preawakening EEG interval. However, the postawakening interval and the stite changes between sleeping and waking, described in EEG terms, may also be important in moderating the retrieval of the dreamed experience. This study will examine these possibilities by evaluating the EEG at postawakening and preawakening to postawakening time intervals.

Chapter One presents a historical review of the main theoretical and empirical research literature linking EEG brain electrical activity to dream recall. Of the various models explaining dream recall performance (Cohen, 1979; Goodenough, 1978), only the ones associating cortical arousal, as measured by the EEG, to dream recall will be reviewed. The empirical research review includes both studies using sleep stage scoring and computer techniques to define cortical arousal. Chapter Two presents a research study using period analysis techniques instead of spectral analysis to characterize the EEG, to verify claims by Koukkou and Lehmann of the dependency of recall on EEG-defined activation states. Typically, the relationship between dream recall and cortical activation has been examined using EEG variables considered individually. However, the question remains whether the association between recall and cortical 
activation nay be described as a linear combination of EEG variables. In this study, both univariate and multivariate approaches were used in an attempt to distinguish the recall success and failure groups. Chapter Three provides discussion and implications generated by the findings in Chapter Two. 
CHAPTER ONE

1.1. Sleep stage EEG and dream recall: Theory and research

In 1953, Aserinsky and Kleitman published the first paper relating dream recall to a particular type of cortical activity during sleep. Their study indicated a high incidence of dream recall in subjects awakened during periods of low voltage, mixed frequency EEG activity accompanied by intermittent bursts of rapid eye movements (REM). Awakenings from periods of sleep other than this activated state produced little or no recall of dreams. These findings were confirmed by Dement and Kleitman (1957b) who found $80 \%$ dream recall following REM awakenings as compared to $7 \%$ recall following awakenings from non rapid eye movement periods (NREM). Their criterion of successful dream recall or dreaming, consisted of a fairly detailed description of the dream content by the subject at the time of awakening. Claims of dreaming without recall of content or vague, fragmentary recollections of content were considered dream recall failures. By these latter definitions, the presence of rapid eye movements during light sleep (stage 1) was established as the criterion determining the occurrence of dreaming, while NREM sleep (stages $2,3,4$ ) became associated with nondreaming.

The strong association between high levels of EEG activation and high recall rates was emphasized as awakenings made during NREM sleep shortly after the termination of a REM period greatly reduced the amourst of recall (Dement \& Kleitman, 1957b; Foulkes, 1966; Goodenough, Lewis, Shapiro, Jaret \& Sleser, 1965; Kamiya, 1961; Wolpert \& Trosman, 1958). Furthermore, it was noted that 
REM sleep may have arousing effects on NREM recall. Evidence indicated that as the interval between the end of the REM period and the time of awakening increased (up to 20 minutes), the recall became more fragmented and failure to recall increased (Wolpert \& Trosman, 1958). Therefore, the few dream reports obtained from NREM awakenings were considered to be the recall of dreams experienced in the preceding REM periods or the recall of thoughts experienced while falling asleep (Dement \& Kleitman, 1957b; Goodenough, Shapiro, Holden \& Steinschriber, 1959). Generally, the presence of spindles and K-complexes with low voltage EEG activity defining stage 2 sleep and/or the predominance of high amplitude delta EEG activity characterizing stages 3 and 4 sleep were considered incompatible with dreaming (Dement \& Kleitman, 1957a). However, Baldridge, Whitman \& Kramer (cited in Herman, Ellman \& Roffwarg, 1978) reported that after the termination of the prior REM period, dream recall decreased but subsequently improved with time spent in NREM sleep (between 30 to 60 minutes). These findings suggested that NREM dream reports may not represent the recall of preceding REM sleep experiences.

Later research by Goodenough et al. (1959) and Kamiya (1961) produced NREM dream recall rates of $35 \%$ and $46 \%$ respectively, indicating that the incidence of recall in NREM sleep may be greater than had been previously reported. The issue of NREM dreaming was more specifically addressed by Foulkes (1962). Defining recall as the report of 'some item of specific content', Foulkes (1962, p.17) found $74 \%$ of awakenings from NREM sleep produced dream reports. 
When claims of dreaming without content were included, recall rates increased to $87 \%$ for NREM sleep relative to $92 \%$ for REM sleep.

The discrepancy in recall rates between Foulkes' (1962) study and earlier reports may be accounted for by methodological differences such as subject sampling, interview technique, awakening procedure and recall criterion (Foulkes, 1966; Herman et al., 1978). Nevertheless, the findings by Foulkes (1962) indicated that the exclusive association of dreaming to REM sleep was no longer tenable and suggested that dreaming, depending on how it was defined, may be continuous throughout the night in all sleep stages.

In addition to dream reports being elicited prior to the occurrence of the first REM period, the growing number of qualitative distinctions found between the content of REM and NREM reports dispelled earlier notions of NREM reports representing the recall of prior REM experiences (Foulkes, 1962; 1966; Rechtschaffen, Verdone \& Wheaton, 1963; Verdone, 1965). Similarly, later assumptions that NREM reports were experiences formulated in the process of waking up (hypnopompic artifacts), while falling asleep (hypnagogic phenomena) or confabulations aimed at pleasing the experimenter were equally disproved (Fiss, 1969; Goodenough et al., 1965; Pivik \& Foulkes, 1968; Rechtschaffen et al., 1963). Both the occurrence and validity of NREM reports as recollections of preawakening NREM sleep experiences have now been firmly established (Cohen, 1979; Foulkes, 1985; Herman et al., 1978).

It is now widely recognized that dreaming or dreamlike experiences occur in a variety of states; in all stages of sleep 
including sleep onset (Foulkes, 1962, 1985; Foulkes \& Vogel, 1965; Herman et al., 1978; Vogel, 1978), during daytime naps (Slap, 1977), and even during wakefulness (Antrobus, 1986; Foulkes, 1985;

Foulkes \& Fleisher, 1975).

However, a considerable amount of research indicated marked physiological and psychological differences between the REM and NREM sleep states (Schwartz, Weinstein \& Arkin, 1978; Verdone, 1965). REM sleep in comparison to NREM was characterized by high levels of activation in EEG and in a variety of physiological systems (respiratory and cardiovascular systems) (Rechtschaffen, 1973; Snyder, 1971; Spear \& Gordon, 1981). Correspondingly, there was consistently more recall produced on awakenings from REM sleep than on awakenings from NREM sleep (Pivik, 1978). As well, the reports obtained from NREM sleep were generally described as poorer, less dreamlike, more thoughtlike, briefer and less emotional than reports from REM sleep (Rechtschaffen et al., 1963). Thus, stages of sleep with low amplitude, fast frequency, desynchronized EEG activity such as REM and NREM stage 1 sleep became associated with high incidence and better quality recall, while stages with synchronized, high amplitude, slower EEG activity such as found in stages 2,3 or 4 sleep were associated with poor recall and less elaborated dream reports (Foulkes, 1966; Rechtschaffen et al., 1963). A number of researchers (Foulkes, 1966; Wolpert, 1972; Goodenoingh, 1978) linked the difficulty to recall dreams out of NREM sleep awakenings to the lack of consolidation of the memory trace in long term memory (LTM) for those stages of sleep. It was assumed that 
the transfer of information from short term memory (STM) to LTM was less effective as the level of cortical arousal decreased. It followed that the necessary filing, coding and reorganization of information through the STM necessary for the transfer to LTM was impaired. Consequently, the accessibility to the dream experience was reduced. Unless the dreamer was awakened during or soon after the dream experience to retain some cue for retrieval, then recall was difficult or impossible. Therefore, time spent focusing on the experience after awakening would increase recall and conversely, distractions at awakening would hinder the transfer of the limited capacity STM to the LTM stores (Cohen, 1979; Koulack \& Goodenough, 1976; Goodenough, 1978).

The major restricting factor in linking EEG-defined activation with recall was the characterization of NREM sleep as one global state. Combining stages 2,3 and 4 into one NREM state did not allow the examination of differences among those sleep stages defined by different EEG patterns. Thus, the association between dream recall and corical arousal was further examined by Pivik and Foulkes (1968) within the NREM sleep state. Their findings indicated that the incidence of recall decreased as sleep deepened. For stages 2, 3 and 4 recall values totaled $71.6 \%, 64.3 \%$ and $46.4 \%$, respectively. In addition, recall rates were lower early in the night as compared to later in the night. That is, there was an increase in the incidence of recall from the first to the last or fourth awakening (45\% vs $73.7 \%$, respectively). Thus, as NREM early in the night consists predominantly of stages 3 and 4 deep sleep, and NREM late in the 
night is dominated by light stage 2 sleep, recall values increased correspondingly with the increasing EEG activity level. As a result, Pivik and Foulkes (1968) proposed a linear relationship between the 'intensity of mental experience and electrophysiological arousal' ( $p$. 147).

Their proposal was an attempt to explain both changes in the quantity and quality of recall. However, their findings did not fully support the proposed relationship between dream recall rates and EEG arousal. As noted by Pivik and Foulkes (1968) recall values varied somewhat independently of sleep stage on the initial awakening of the night. These results may in part be attributed to the unequal distribution of awakenings across sleep stages. There was a predominance of awakenings from stage 4 sleep (predominance of delta sleep) with virtually no awakenings from stage 2 sleep on the first awakening of the night, with a reversed trend for the following awakenings. The results noted by Pivik and Foulkes (1968) confounded sleep stage with time of night effect (Schwartz et al., 1978).

Later research by Tracy and Tracy (1974), using a large number of awakenings and comparing recall rates of awakenings taken at corresponding points in time (i.e. holding the time of night constant) found no significant differences between the amount of recall for stage 2 sleep relative to stage 4 sleep (stage $2=54 \%$; stage $4=50 \%$ ). Their study did not control for the proximity to REM effect, possibly a major influence on NREM recall rates (Dement \& Kleitman, 1957b; Foulkes, 1966; Goodenough ei al., 1965). However, the 
findings by Tracy and Tracy (1974) cast some doubt on the validity of Pivik and Foulkes' (1968) linear EEG-defined cortical arousal model as it relates to quantity of dream recall. Unfortunately, Tracy and Tracy (1974) did not report the number of failures to recall from the different stages of sleep. There appears to be approximately 100 observations not included in their study. In addition, information is lacking about the number of subjects used in their within subject analysis. Therefore, these limitations weaken Tracy and Tracy's conclusions regarding the lack of relationship between dream recall and EEG-defined cortical arousal.

Overall, studies relating EEG to dream recall produced inconsistent findings, the association between the two being quite limited (Pivik \& Foulkes, 1968; Rechtschaffen, 1973). Within NREM sleep, very different EEG patterns described in terms of stages produced very similar amounts of recall. The EEG of stage 2 relative to stages 3 and 4 is quite dissimilar, yet the amount of recall elicited on awakenings from stage 2 and stage 3 are quite similar (Foulkes, 1962; Pivik \& Foulkes, 1968), and possibly those between stages 2 and 4 (Tracy \& Tracy, 1974). There is also a great amount of variation in the incidence of recall reported on awakenings within the same stage of sleep. At present, the frequency of dream recall from stage 2 has been found to vary between $7 \%$ and $74 \%$ (Foulkes, 1962; Herman et al., 1978; Kamiya, 1961; Pivik \& Foulkes, 1968). For example, Pivik and Foulkes (1968) reported $71.6 \%$ of awakenings from stage 2 sleep produced recall leaving $28.4 \%$ of awakenings from the same stage producing no recall. Similarly, recall values for REM 
sleep may vary on average between $64 \%$ and 100\% (Foulkes, 1962).

In sum, awakenings from the same EEG-defined sleep stage may result in different rates of recall, while awakenings from different EEG-defined sleep stages may produce similar amounts of recall.

The previous review dealt with studies investigating the tonic EEG patterns of sleep in relation to dream recall. Another line of research examined the relationship between episodes of phasic EEG activation and dream recall performance. Generally, tonic EEG activity refers to long lasting or sustained sleep events (i.e. sleep stages) while phasic activity refers to episodic, transient EEG events superimposed on the tonic background activity (K-complexes, spindles, theta bursts, etc.). Two other physiological indices, eye movements and electromyographic activity during sleep will be examined. These last two measures are included as they constitute standard indicators in visual sleep stage scoring according to the criteria of Rechtschaffen and Kales (1968). A comprehensive review of the literature by Pivik (1978) indicated that within REM sleep, the presence of eye movements increased the likelihood of recall. However, the differences in the incidence of dream recall between between phasic (high amounts of eye movements) and tonic (low amounts of eye movements) conditions were not large. In agreement with these findings, a recent study by Meir (1989) examined dream recall following tonic and phasic REM awakenings. Meir's study demonstrated that the categories of failure to recall, contentless recall and successful recall could not be differentiated along the 
phasic-tonic eye movement dichotomy. Within NREM sleep, the results of studies examining the effects of $\mathrm{K}$-complexes, sleep spindies in stage 2 (Pivik, Halper \& Dement, 1969; Weisz, 1972) and theta bursts at sleep onset (Pope cited in Pivik, 1978) on dream recall were nonsignificant. Only one study, that of Larson and Fnulkes (1969) investigated submental EMG suppression (lowered chin muscle tonus) in relation to dream recall. It was hypothesized that much like REM sleep, the occurrence of diminished muscle tone during NREM stage 2 sleep would be associated with increased recall. Comparing periods of high EMG with low EMG levels in NREM sleep, their findings indicated lesser, rather than greater, recall with low EMG awakenings. However, the differences in recall frequency between the high and low EMG caiegories were not substantial and in the majority of cases failed to reach statistical significance. Generally, their study suggested that EMG activation in NREM sleep may not be predictive of dream recall performance. In sum, there is evidence to suggest that phasic components of sleep probably do not affect dream recall.

Taken together, studies using measures of tonic and phasic EEG revealed that the simple linear cortical arousal model proposed by Pivik and Foulkes (1968) could not account for the variations in dream recall performance within or across sleep stages. Yet, the strong relationship between high recall rates and the REM state, as well as the nearly invariably higher incidence of recall from REM sleep in comparison to NREM sleep generally suggested that a more consistent association between EEG-defined cortical activation and 
dream recall performance may yet be uncovered. The major limiting factur in describing this association concerns the use of sleep stages to characterize cortical arousal levels. The conventional visual stage scoring method defines a sleep stage as a distinct and invariant pattern of electrophysiological activity. Yet, there is ample evidence of within stage variations in EEG activity across the night (Church et al., 1975; Fein, Floyd \& Feinberg, 1981). The advent of techniques of computer quantification in sleep analysis provides a more detailed evaluation of EEG characteristics then was permitted using stage scoring (Moffitt \& Hoffmann, 1987). This more detailed examination of the EEG may help to better define the relationship between dream recall and EEG activity.

\subsection{Computer-quantified EEG variatles and dream recall:}

Theoretical models

An alternative model relating cortical activity to dream recall processes is that of Koukkou and Lehmann $(1980 ; 1983)$ who presented a functional state-shift model to explain the processing of information during waking and sleeping. This model proposed that dream recall performance is based on a state-dependent principle of learning and retrieval (Lehmann \& Koukkou, 1984; Overton, 1978; Weingartner, 1978) whereby the encoding in memory of information in a given functional state (e.g. sleeping) makes its retrieval difficult in a different state (e.g. waking). However, the degree to which the retrieval of information will be impaired depends on the magnitude of differences between the two states (encoding and retrieval); the greater the similarity of states, the greater the availability for 
retrieval from memory.

Koukkou and Lehmann (1980) defined a functional state as a particular organization of neural activity representing a certain mode of information-processing (cognitive and emotional) which is directly reflected in EEG measurements. The functional state may be widespread and long lasting, such as characterized by a stage of sleep or wakefulness, or it may correspond to brief or short lasting EEG patterns enduring for a few minutes, seconds or less, such as inputevoked responses. It is important to note that the EEG-defined state may correspond to the EEG sleep stage but is not equivalent to it. The EEG state is comprised of continuous fluctuations of brain information-processing states represented by brief series of EEG waves of a particular type (e.g. sleep spindles, K-complexes, waveforms of any frequency such as alpha, beta, delta, etc.). Therefore a given stage of sleep may include a variety of EEG patterns or states.

As reflected in the EEG, each functional state corresponds to a specific long term memory storage space with its specific knowledge set and information-processing strategies. According to Koukkou and Lehmann (1983; in press) the memory system may be described as a hypothetical graded memory storage continuum of low to high activation states. The higher states are associated with EEG patterns closer to wakefulness and resemble the 'reality-adapting', more complex and advanced cognitive-emotional strategies of adulthood functioning. The lower order states are reflected in a lower activated EEG and depict patterns similar to those of early childhood 
developmental stages. Relative to an adult's waking EEG, lower memory storage spaces or states thus represent less developed kinds of knowledge with their more 'childlike' strategies (imagery-fantasy treatment of information, disregard for reality-testing or reflection). At any given moment in time, only a portion of the total long term memory content is accessible for current informationprocessing. The activated contents of the currently accessible long term memory store are held in the working memory. Dreaming is a synthesis of information processed within the working memory system and its associated long term memory storage space (Koukkou \& Lehmann, in press). Following a state-dependent or state-specific mode of functioning, it is assumed that a given state will allow optimal storage and recall of information associated with that particular state. However, according to the asymmetry of statedependent recall, the storage spaces of higher order or more activated states but not of lower order states (except for the immediately neighbouring lower state) may also be partially accessible for recall.

Therefore, the different functional states allow access to different kinds of knowledge, different information-processing strategies as they are associated with different state-specific memory states. Knowledge acquired in a higher activation EEG state or in a state closer to adult wakefulness will remain accessible for recall when lower order less developed states are open, but there will be little recall in wakefulness for information encoded in low arcusal states. The accessibility of information acquired in a lower 
state of arousal will depend upon the similarity of EEG between the encoding and retrieval states; the greater the similarity in EEG between states, the greater the recall. The memory-specific states with their corresponding EEG serve as retrieval cues opening and closing memory storages, activating or disactivating memory contents with corresponding changes in the brain's functional states. Therefore, the functional state defines the 'size' of memory, that is, which memory storages are accessible or which portions of the individual's total knowledge can be used for processing.

Using these definitions, the state-specific relationship between encoding and dream recall may be described as follows: First, as the cortical EEG patterns associated with experiences stored in any sleep state become increasingly similar to those observed in wakefulness, the greater the recall of these experiences upon waking. More specifically, both the degree and duration of EEG activation during encoding affect the transfer of information from sleeping to waking. That is, the higher and longer the EEG activation (expressed by an increase in higher frequency EEG activity or a decrease in lower frequency delta and theta activity) during dreaming, the better the recall during wakefulness. This applies to recall out of REM and NREM sleep. More generally, the transfer of information from sleeping to waking may be expressed in terms of the magnitude of difference between the EEG dreaming and waking states or between the preawakening and postawakening EEG intervals. As the difference in EEG between the two states increases, it is expected that recall performance will decrease. 
Therefore, as stated by Lehmann (1980) and in agreement with the linear cortical activation model (Pivik \& Foulkes, 1968), the dependency of wakeful recall on the quality of sleep EEG during encoding would account for the better recall out of a highly activated state such as REM relative to the impaired recall obtained out of the less activated NREM states, stages 2,3 or 4 sleep. Likewise, subjects awakened early into a REM period should produce less recall than subjects awakened a lengthier period of time after REM onset. Such proposals are in line with the earlier notion that the level of arousal at or very close to the time of encoding of the dream event was a critical factor in explaining dream recall failure (Koukkou \& Lehmann, 1968; Koulack \& Goodenough, 1976).

Secondly, according to Koukkou and Lehmann (1983), the momentary state shifts in the direction of higher EEG arousal during dreaming may account for the correspondingly high or low incidence of recall within any given sleep stage. Incoming information, judged novel or meaningful by the logic processing system with its short term memory triggers an automatic change in the functional state through the orienting response, leading at once to increased frequency brain electrical activity. This state-shift is believed to reflect the shift in attention necessary to process material in a new higher functional state (Lehmann, 1990). The change in state closes the memory storages of the lower arousal states and thereby restricts the retrieval of information that has been acquired in that state. At the same time, the state-shift implies a switch to a different higher memory storage space with its associated more 
advanced cognitive strategies and responses. These brief shifts of brain state facilitate or hamper dream recall by altering the magnitude of difference in states between storage of the dream experience and its retrieval. Thus, during encoding, there may be instances of heightened EEG arousal, inducing a functional state sufficiently close to wakefulness to increase dreain recall upon awakening. However, the orienting response may be too large and lead to an exceedingly high level of arousal upon awakening. By this process, the distance between the sleeping and waking states is increased and results in diminished dream recall. While recounting the dream in wakefulness, additional orienting responses to the dream content may further increase the EEG, thereby further restricting access to the memory space where the dream was stored prior to the state change. This may produce partial or no recall of the dream experience. Two factors thought to induce state-shifts because they modify arousal levels (Koukkou \& Lehmann, 1983) are the salience of the dream (novelty, bizarreness, vividness) during sleep, and an interference factor upon awakening (distractions) (Koulack \& Goodenough, 1976; Cohen, 1974, 1979; Goodenough, 1978).

Thirdly, Koukkou and Lehmann (1980) proposed that previously stored dreams, forgotten upon awakening, may become re-activated and available for recall given the reinstitution of the initial encoding EEG state. The familiar experience of forgetting a dream at the moment of awakening and recalling it some time later supports the notion of recall failures as a retrieval rather than as an 
encoding or consolidation problem. Goodenough (1978) has reported that recall is improved if attention is focused on the dream experience for a short while after awakening. However, there is no research known to this author which has examined the relationship of delayed dream recall and EEG states prior to or following awakening.

Within the framework of this model, dreaming is continuous throughout the night, like information-processing during wakefulness. It involves the same brain mechanisms in recognizing and evaluating the current internal and external realities of the individual and synthesizing the information into an appropriate response, the dream. The different EEG activation levels do not alter the capacity of information-processing, rather they determine the type of knowledge and strategies available for processing. According to Koukkou and I,ehmann (1983), there is no difficulty of encoding or storage during sleep, rather dream recall failure is experienced as a problem of retrieval upon awakening.

Unlike Koukkou and Lehmann (1983) who described dream recall strictly as a problem of retrieval, Antrobus (1986) proposed that dream recall was dependent on the overall level of cortical activation as it affects either dream production, memory or retrieval processes (Wollman \& Antrobus, 1987). More specifically, it is the level of activation of the working memory system which would allow for the recall of dreams generally. By definition, the working memory contains all information from the long term declarative memory (LTM) that is currently active and accessible to the dream 
production system. The information is organized in cognitive units which are interconnected to form a complex neural network allowing activation to spread from one unit to the other. The continuous flow of activation between related units would ensure that the dream remains temporarily stored (consolidation) and accessible in working memory or that a sufficient number of elements remain active to engage production or to permit later retrieval. It is expected that the amount of dream recall increases with increasing levels of cerebral arousal. A reduced state of cerebral arousal may decrease the capacity of working memory (restrict the availability of mnemonic sources from LTM for the process of dream production) thereby diminishing the number of active units necessary to activate other related units into constructing a dream sequence. A limited storage of information in working memory may also restrict the ability to retrieve a dream sequence as less information units remain active to be used as cues to access the remainder of the dream episode.

Generally, as stated by Antrobus (1986) the level of cortical activation varies with the biological state such as sleep stages. The less activated states, stages 2,3 , or 4 , would show correspondingly less recall given their smaller size working memory, whereas higher activated states such as REM sleep would be associated with greater recall rates. Aside from the theoretical reformulation, the proposed relationship between recall and EEG-defined arousal is in agreement with that of Koukkou and Lehmann (1983) and Pivik and Foulkes (1968). According to Antrobus (1986), increments in cognitive activation (working memory) are reflected in overall cortical arousal 
and may be evidenced in EEG power spectrum measures.

The physiological processes underlying the initiation of REM sleep were described by McCarley and Hobson (1975) in their reciprocal interaction model. These processes serve as the basis of the activation synthesis hypothesis (Hobson and McCarley, 1977; Hobson, 1988) to account for dream production and dream forgetting. The cortical/neural activation model presented by Hobson (1988) states that REM sleep and dreaming are the consequences of a continuous reciprocal inhibition and excitation of two interconnected neuronal populations in the brain stem. Basically, as the inhibitory or REM off aminergic neurons of the pontine brain stem become deactivated and reach a critical low level of activity, the excitatory or REM on cholinergic neurons of the reticular formation become active and the brain actirity is switched to a REM sleep dreaming mode. Although this reciprocity of activity is assumed to be continuous across the waking, NREM and REM states (Hobson, Lydic \& Boghdyan, 1986), the relation to dream recall is applied only to REM sleep.

The aminergic neurons are found in the brain stem in three locations 1) the noradrenergic locus coeruleus (LC) 2) the serotonergic raphe nuclei and 3) the peribrachial pons (beside the LC). The greatest proportion of REM off cells is found in the dorsal raphe nuclei. A few have also been found throughout the midbrain reticular formation. The REM on cells are contained in the reticular formation. The inhibitory effect of the aminergic neurons affect the entire brain because of their wide distribution of axons extending throughout the forebrain, into the frontal lobes and across the cortex, 
as well as in the spinal cord. As such, they are considered the modulatory or state regulatory neurons determining the metabolic mode of the brain (Hobson, 1988). In REM, the failure to recall a dream is intimately linked to the activity of these modulatory neurons. When the aminergic neurons are turned off, the forebrain circuits are activated to mediate the dream experience without encoding it in long term memory. According to Hobson (1988), the dreamed events are temporarily stored in short term memory and are accessible only if the dreamer is awakened during the dream experience as the modulatory neurons are reactivated. The increased activity of aminergic neurons allows the consolidation of the dream in long term memory. Therefore, the processes underlying encoding and storage in long term memory are not functional during REM sleep due to the cessation of aminergic neuronal firing (McCarley, 1981) and would account for the failure to recall dreams. According to Hobson (1988), REM sleep and dreaming specifically, occur only when the aminergic neurons are deactivated.

In line with Hobson's view, Steriade and McCarley (1990) recently proposed that NREM dreaming and dream recall may occur only when brain neuronal activity approaches levels recorded in REM sleep but fails to reach full intensity. Dream experiences outside of REM sleep are described inaccurately as "rambling, obsessive, and purely verbal ruminations" (p.417). They identified the sleep period immediately preceding the onset of REM sleep as a moment of intensification of cerebral arousal and as the most likely to promote vivid dreaming and subsequent NREM dream recall. Generally, the 
interval of stage 2 sleep just prior to the onset of REM sleep (30 to 60 seconds) is associated with a sharp decrease in muscle tone (EMG) to levels recorded in REM sleep. The EMG suppression in NREM sleep precedes the appearance of EEG and EOG (electro-oculographic) events defining the onset of REM sleep. As suggested by Hobson (1988) and Steriade and McCarley (1990), the sleep interval showing increased cerebral arousal together with a sharp decline in muscle tone just prior to REM onset should result in more vivid dreaming and greater rates of dream recall. Larson and Foulkes (1969) have systematically examined the high (prior to EMG suppression) and low EMG (signalling REM onset) conditions for stage 2 awakenings just prior to REM occurrences in relation to dream recall. Their results indicated both lower recall with EMG suppression prior to REM rather than the expected REM-like higher recall and no differences between the high and low EMG conditions in dreaming quality. Therefore, there appears to be no eviderice to support Steriade and McCarley's (1990) notion of a NREM dream and recall process activated solely by its proximity to REM onset. The activation synthesis model is specific to REM sleep and in this manner does not account for NREM dreaming.

\subsection{Computer-quantified EEG variables and dream recall:} Empirical research

Following the theoretical proposals of Koukkou and Lehmann (1980) and Antrobus (1986), six recent studies using computer analyses to evaluate EEG characteristics have examined the relationship between cortical EEG activation and dream recall. 
Briefly, the two major computer techniques used to analyze the electrophysiological sleep data in the studies to be reviewed are spectral analysis and period analysis. Spectral analysis provides one measure of the intensity of the EEG activity indexed in terms of power. Spectral analysis evaluates power in terms of amplitude and frequency and therefore does not allow for the evaluation of the unique contribution of these components. Period analysis yields a power measure and two measures indicating the amount of time spent in a given frequency category: percent zero cross and percent first derivative. Unilike spectral analysis, the period analysis algorithm used in the present experiment examines time in frequency independent of the amplitude (or power) for a given frequency event. The first derivative analysis preferentially quantifies the fast frequency EEG variations superimposed on the slow frequency activity. For both techniques, power measures may be computed for frequency categories of interest or averaged across frequeacies to provide an overall power measure. The power measures provided by period analysis are highly correlated with those of spectral analysis, the two techniques offering comparable information (Pigeau, Hoffmann, \& Moffitt, 1981).

Lehmann, Dummermuth, Lange \& Meier (1981) examined the differences in EEG between REM periods followed by dream recall and REM periods without dream recall. They failed to provide the definition used to assess dream recall. Spectral power analysis was applied to the 20 second preawakening EEG segments. Of 28 awakenings, $61 \%$ were associated with recall and $39 \%$ with no recall. 
A between subjects analysis indicated that awakenings with recall relative to no recall, were significantly associated with less power in all frequencies recorded (from $.4 \mathrm{~Hz}$ to $14 \mathrm{~Hz}$ ). This finding was most significant in the left parietal-vertex electrode placement. In addition, REM periods without recall were shorter than REM periods with recall. However power values did not systematically vary with changes in the duration of the REM periods. This last finding would appear not to support Koukkou and Lehmann's (1983) proposal that the longer the time spent in an activated state such as REM, the better the dream recall.

Using spectral analysis, Antrobus, Ehrlichman, Wiener \& Wollman (1983) computed the total EEG power value for the 3 minute interval preceding each of 42 REM sleep awakenings. The high and low power EEG periods were examined in association with the corresponding dream reports scored on the total recall word count measure (TRC) and four visual imagery scales (visual nouns; visual modifiers (visual adjectives and adverbs); spatial relations (prepositions); visual action verbs). The TRC measure was defined as the number of words used by the subject to describe his preawakening sleep experiences, excluding commentaries, pauses, corrections and repetitions. Unlike the total word frequency measure (the count of all words in a report) which represents speech fluency, total recall count is assumed to strictly reflect the recall of information. Antrobus (1983) found that the total recall word count measure was the best variable discriminating REM from NREM stage 2 sleep reports, accounting for all qualitative differences 
between the two states. This quantitative difference in length of report between REM and NREM sleep was assumed to be dependent on the general level of cortical activation (Antrobus et al., 1983). Thus, the greater the level of cerebral arousal, the greater the amount recalled.

However, findings from Antrobus et al.'s study (1983) indicated that for a single 2 to $12 \mathrm{~Hz}$ EEG frequency band, neither total recall count nor any of the imagery scales showed any distinctive association with the level of cortical activation as indexed in the overall spectral power measure. In addition, the examination of trends in EEG activation within the 3 minute preawakening interval failed to produce any correspondence between activation levels and the total recall count and imagery measures.

These findings are congruent with those of Wollman and Antrobus (1987) who examined the EEG data of 30 subjects, 17 males and 13 females, awakened at predetermined intervals out of REM sleep. The EEG recordings were taken at central and tempoparietal sites. The absolute spectral power was calculated for each of six frequency bands (delta, theta, alpha, sigma, beta and above $30 \mathrm{~Hz}$ ) for the 1 minute and 5 minute preawakening periods for each subject. The EEG measures were then related to corresponding dream reports scored on the total recall count measure, four scales measuring visual imagery, two scales measuring explicit and implicit speech and a thought unit scale (a distinct thematically homogeneous thought sequence). The study also included a waking condition. It was hypothesized that the quantitative differences in recall of 
content would vary as a function of cortical activation. Results indicated that total recall count was not significantly associated with any of the EEG power variables examined during REM. Visual modifiers were negatively related with delta and sigma power during the 5 minute and 1 minute REM periods. The thought units were positively related with delta and theta power for the averaged 5 minute EEG period only. Total recall count, as an index of the activation of a verbal or more global cognitive production system (Wollman \& Antrobus, 1987) would appear not to be associated with corresponding changes in overall EEG cortical activation. If, within the context of a successful recall, increases in TRC reflects an increase in recall performance, these findings would appear not to support Koukkou and Lehmann's state-dependency hypothesis. However, specific components of the TRC variable may vary with activation levels.

Another study, that of Meier (1989), investigated the differences in mean spectral values between reports divided with respect to recall success or failure, into categories of dreams with content, dreams without content (definite feeling of having dreamt with no content recalled) and no dreams (failure to recall aray experiences). The study comprised 7 males and 3 females selfreported low dream recallers (at least one recall per month) studied over 4 consecutive nights. The 16 second preawakening EEG segments of subjects awakened from REM sleep ( 5 minutes after each REM onset) were examined. The EEG recordings were taken at parietal and tempoparietal sites. Of a total 160 awakenings, there 
were $63.8 \%$ dreams $(n=102), 21.3 \%(n=34)$ no dreams and $15 \%(n=24)$ no content recall. In agreement with Lehmann et al. (1981), recall increased with decreasing mean power values as evidenced throughout the whole frequency spectrum $(2 \mathrm{~Hz}$ to $25 \mathrm{~Hz}$ ). Power values were highest in the no dream category and lowest in the dream category. There were intermediate scores for the no content category. The dream and no dream categories were significantly different on all EEG measures, the dream and no content categories differed occasionally, while the no content and no dream categories showed very few significant differences. Due to the high variability in recall between individuals (from $14.3 \%$ to $93.8 \%$ ), the differences between recall categories were eliminated when comparisons across recall groups were made within subjects rather than between subjects. The within group analysis suggests that recall group differences reported using the between subject analysis were probably due to subject variability and could not be accounted for by EEG variations. In sum, for REM sleep, the studies by Lehmann et al., (1981) and Meir (1989) cannot be regarded as conclusive evidence supporting the proposed state-dependent cortical activation model of Koukkou and Lehmann. The use of low dream recallers has been shown to be problematic in dream recall studies (Armitage, 1986; Purcell, Mullington, Moffitt, Hoffmann \& Pigeau, 1986) because of the large within group variability in response to experimental procedures, which may affect recall performance. Apparently, the low recall rates (64\%) reported from REM sleep in Meier's (1989) study and the large intersubject variability in recall may be 
attributed to the selection of that subject population.

Two studies investigated the relationship between EEG-defined cortical arousal and dream recall in NREM sleep. Using period analysis, Moffitt et al. (1982) examined the 5 minute averaged preawakening tonic EEG data of subjects awakened from REM and stage 2 sleep. The EEG recordings were taken at central sites. The subjects were 8 right handed males, four high frequency (5 dreams a week or more) and four low frequency (one dream per month or less) self-reported home dream recallers. Successful recall was defined as the description of at least a single image, feeling or thought attributed by the subject to the preawakening interval. Results indicated that there were significant interactions between recall and subject type. Nonrecall was associated with lesser amounts of sigma zero cross and greater amounts of power and percent time in the delta frequency band for the high dream recallers relative to low dream recallers. When examined in relationship to an averaged 4 minute postawakening rehearsal interval, Moffitt et al. (1982) found no significant differences in recall in association with sleep stage. Thus, the combined postawakening REM and stage 2 EEG revealed that successful recall relative to no recall, was associated with more eye movements and more alpha frequency activity (alpha first derivative measure). In a study by Williamson, Csima, Galin \& Mamelak (1986) six subjects ( 3 males, 3 females) studied for four nonconsecutive nights were awakened at predetermined inter als throughout the night, following an accumulated five minutes into stage 2 sleep. Each 32 
second preawakening EEG periods for the 76 awakenings were subjected to power spectral analysis and examined within subjects, in association to the reports rated on the Foulkes' dreamlike fantasy scale (dreaming, imagery, thinking and no recall categories). The recorded EEG samples we:e from frontal and central placements and examined in three frequency bands: Delta-theta, alpha and sigmabeta.

Overall, Williamson et al. (1986) found that with increasing dream recall there was a corresponding increase in mean frequency in the sigma-beta band on the right side of the brain, with a similar nonsignificant trend recorded on the left side of the brain. On that measure, the no recall category was significantly different from the thinking and imagery categories, but the no recall and dreaming categories were not significantly different. In addition, there were no significant differences between the recall groups on any power measures, nor on any of the power measures comparing the first half to the second half of the night. In agreement with Moffitt et al. (1982), Williamson et al. (1986) reported that power in the thetadelta bands within stage 2 tended to fall with increasing recall, however this trend failed to reach statistical significance.

In sum, with respect to preawakening EEG, there is evidence to suggest that successful dream recall may be associated with higher cortical activation as reflected either in lower total power values in REM sleep (Lehmann et al., 1981; Meier, 1989), and greater amounts of sigma zero cross, lower amounts of delta zero cross and power for high dream recallers (Moffitt et al., 1982) or increased mean 
frequency of the sigma-beta band for stage 2 sleep (Williamson et al., 1986) in accordance with Koukkou and Lehmanns' (1983) statedependency proposal. However, these findings are clearly not uniform across studies or across states, REM and NREM (stage 2 , specifically) and thus warrant further investigation. Only one study, that of Moffitt et al. (1982), investigated the postawakening EEG in relation to dream recall. Since their study revealed no significant differences in recall in association with sleep stage, their findings were reported for the combined stage 2 and REM sleep intervals. The postawakening EEG interval constirutes a critical time period for the transfer of information from sleeping to waking, and for that reason should be more closely examined.

1.4. Hemispheric EEG variables and dream recall: Theoretical issues

Other electrophysiological indices of cortical activation examined in conjunction with dream recall are the hemispheric asymmetry measures. The theoretical basis for EEG hemispheric activation studies originates in neurophysiological and behavioral research involving normal subjects, partial and fully commisurotomized patients (individuals with interhemispheric connections severed), and brain lesioned individuals (Bryden, 1982; Eccles, 1977; Springer \& Deutsch, 1989, for full review). Generally, for right-handed or left hemisphere dominant individuals, the right hemisphere during wakefulness has been described as nonverbal, the expressor of unconscious processes and reactions (Eccles, 1977; Galin, 1974) involved in integrative, visuospatial and holistic functions (Boisacq-Schepens, 1983). The left hemisphere has been 
viewed as symbolic and analytical, sequential and propositional, specialized in verbal and language processes (Eccles, 1977; Bryden, 1982). Left-handed individuals typically show less consistent laterality of neurological organization (Galaburda, LeMay, Kemper \& Geschwind, 1978; Springer \& Deutsch, 1989).

Early research suggested that because of its predominant visuo-imaginal characteristics, the production of dreams in REM sleep should be mediated by right hemisphere processes (Bakan, 1977; Broughton, 1975; Galin, 1974). Although many dream 'reports collected from REM sleep were indistinguishable from those collected from NREM sleep (Foulkes, 1966; 1985), there existed a pervasive notion defining REM experiences as dreams and NREM ones as thoughts. Taking into account the physiological and content differences between REM and NREM dreams, Broughton (1975) further proposed that REM dreaming should be associated with greater right hemisphere activation while the more thought-like, less visual NREM experiences should be associated with greater left hemisphere activation. The left hemisphere was also assumed to be involved in the verbal communication of the dream experience upon awakening. Evidence from a few cases indicating the loss of dream recall for subjects with damage to the right hemisphere, as well as research indicating a small shift in right hemisphere activation during REM sleep, appeared to support the hypothesis of a right hemisphere dreaming process (Bakan, 1977). According to Galin (1974), the specialized mode of functioning of one hemisphere may restrict the translation of information to the other hemisphere. In 
line with these proposals, Antrobus and Ehrlichman (1981) argued that the difficulty to recall dreams may be due to the left hemisphere functioning individual who upon awakening cannot access the dream coded in the right hemisphere visual memory stores. As in state-dependent learning and recall, it was thus expected that dream recall increased as the hemispheric state of the waking individual approached that during dreaming. There should be greater right hemisphere activation associated with increased incidence of recall (Antrobus \& Ehrlichman, 1981).

In contrast to the visual-imaginal right-hemisphere dreaming process, other researchers (Antrobus, 1978; Foulkes, 1978; 1982) believed that dreaming was essentially a thinking process and that the left-hemisphere verbal linguistic system was thus largely responsible for dream formation. The visual-imaginal representations (imagery and affect) of the dreamed experienc: characterized a secondary process organized according to the rules of the propositional, verbal processing system.

Recent studies examining laterality in sleep, with and without awakenings to assess recall, failed to support the association of a strictly right-hemisphere or left-hemisphere REM, and lefthemisphere NREM dreaming process (Ehrlichman, Antrobus, \& Wiener, 1985; Herman et al., 1987; Herman, Rampy, Roffwarg, Homan, \& Hirshkowitz, 1989; Moffitt et al., 1982; Pivik, Bylsma, Busby \& Sawyer, 1982; Rosa, Antrobus \& Reinsel, 1989). Evidence against a strict association of right hemisphere dreaming was provi!led by dream recall studies in fully commisurotomized patients 
(Farah, Gazzaniga, Holtzman \& Kosslyn, 1985; Greenwood, Wilson \& Gazzaniga, 1977). In those studies, patients functioning with the left hemisphere only were still able to report visual dreams. Furthermore, a comprehensive review by Greenberg and Farah (1986) of dreaming-dream recall following brain damage strongly favours the posterior regions of the left hemisphere as critical in producing dream imagery. Yet, loss of dreaming was also reported following both right and left hemisphere damage (Murri et al., 1989; Schanfald, Perlman \& Greenberg, 1987) suggesting a specific contribution by the two hemispheres in the process of dreaming. Recently, Armitage, Hoffmann, Loewy \& Moffitt (1989) found that REM sleep was characterized by interhemispheric balance, stage 2 by small asymmetries and that slow wave sleep (stages 3 and 4 ) showed the highest degree of asymmetry, especially in low frequency categories.

The main problem with these theoretical propositions resides in the failure to distinguish the dream recall from the dream generation process. In fact, the topic of dream recall was not directly. addressed by researchers, the interest being focused on dream production. Dream recall performance was implicitly linked to the dream generation process, and assumed to be facilitated in a linear fashion by the same neural processes.

A few researchers (Antrobus, 1987; Cohen, 1979; Koukkou \& Lehmann, 1983) have suggested that the left hemisphere may be involved in the process of dream recall. Irrespective of where dreaming is generated, the recall of a dream experience may include 
at least two processes, dream-remembering and dream-reporting (Pivik et al., 1982). Dream-remembering may be partly mediated by consciousness of the dreamed experience (Cohen, 1979). On the other hand, dream-reporting may be determined by the ability to retrieve the experience from memory and to produce a verbal report upon awakening (Antrobus, 1987; Cohen, 1979).

In line with these assumptions, dream recall would imply increased left hemisphere involvement for the conscious noticing of dreamed events, as well as the decoding or interpretation of the dreamed experience into a verbal form, and for the actual verbalization in the form of a verbal report (Antrobus, 1987). Therefore, it is assumed that a relative increase in left-hemisphere activation should be associated with a corresponding increase in dream recall generally (Antrobus, 1987; Cohen, 1979; Koukkou \& Lehmann, 1983).

These assumptions apply to the expected relationship between tiv. efficiency of hemispheric functioning and dream recall at the preawakening interval, according to the state-dependent hypothesis. However, regardless of the patterns of asymmetry depicted during sleep, it is expected that the left hemisphere is essential for the verbalization of the dreain report upon awakening (Antrobus, 1987; Koukkou \& Lehtwar, iSc3),

1.5. Hemis heric $\mathrm{EE}$ : Only . Jse stuci:s which have used EEG hemispheric measures obtained during sleep or upon awakening, in association to dream recall, in neurologically intact individuals, will be reviewed. 
Lehmann et al. (1981) reported that coherence analysis (correlation of values from different electrode placements) for REM awakenings showed no systematic relationship with recall. If interhemispheric coherence is taken to be an indication of shared activity between hemispheres, then these findings would suggest no clear laterality effects associated with recall performance. Pivik et al. (1982) using spectral analysis, computed interhemispheric ratio power values for each one-minute preawakening and two minute postawakening REM EEG sleep intervals for each of the 5 conventional frequency bands. These ratio values were then correlated with dream reports rated on the Goodness of Recall 5 point scale, depicting increasing elaboration of recall and a 4 point visual-verbal scale characterizing visuo-spatial and language dimensions. Their measure of relative hemispheric symmetry or balance was expressed as the difference ratio between hemispheres according to the formula $(L-R) /(L+R) \times 100$ where $L$ and $R$ represent values from the left and right hemisphere, respectively. This measure reflects the degree of asymmetry between hemispheres rather than hemispheric right or left dominance. Of a total 55 awakenings, $44(80 \%)$ resulted in the recall of some content. Due to the great variability in scores between subjects, the correlations between recall and EEG measures were generally weak. Overall, for the preawakening interval, their results indicated a nonsignificant trend towards increased left frontal activation in association with increasing amount of dream recall and greater emphasis on the verbal elements of the reports. For the two minute postawakening interval, the correlations between EEG variables and 
recall were minimal across subjects and, for the preawakening period as well, generally nonsignificant within subjects, for both frontal and central recordings. In sum, as evidenced from these two studies, there would appear to be little support for marked left-hemisphere activation with dream recall preceding or following REM sleep awakenings.

Studies investigating the relationship between dream recall and hemispheric lateralization measures obtained from NREM sleep (stage 2) EEG intervals have produced largely negative results. Moffitt et al. (1982) using period-analyzed EEG data, calculated asymmetry ratio difference values $(L-R) /(L+R) \times 100$ between homologous central electrode sites. For the preawakening interval, their findings indicated no significant differences between success and failure of recall on any of the asymmetry EEG measures.

These results are consistent with those of Williamson et al. (1986) who reported no significant relationship between any of Foulkes' dreamlike fantasy scale categories of recall on measures of interhemispheric coherence ratio. Neither the simple ratio of total power between the two hemispheres for averaged frontal and central derivations $(F 4, C 4 / F 3, C 3)$ nor the ratio of mean frequencies revealed any significant relationship with respect to dream recall. Taken together, the results of these studies failed to show any clear pattern of lateralization in relation to dream recall performance.

Using a different approach, Antrobus et al. (1983) examined the preawakening EEG activity recorded from ternporal and parietal electrode sites of subjects awakened to report their dreams, 
following periods of increased right and left hemisphere activation in REM sleep. For each subject, the pair of awakenings showing the greatest hemispheric EEG shift, left to right and right to left, was analyzed in relationship to corresponding reports scored on the word count measure and four imagery word count scales. The EEG activity was combined into a single $2 \mathrm{~Hz}$ to $12 \mathrm{~Hz}$ frequency band. It was $h_{j}$ othesized that if dreaming is mediated by the right hemisphere there would be a higher word count for all scales for the right activated relative to the left hemisphere activated awakenings, especially for the visual scales. Results indicated no differential influence of left relative to right hemisphere activation in association with the total recall count measure. Thus, the magnitude of recall in terms of the number of words reported was not significantly associated with hemisphere predominance. However the visual action (verbs) and spatial relations scales were significantly associated with increasing left hemisphere dominance rather than the expected right hemisphere. A similar but nonsignificant trend was reported on the speech scale (explicit and implicit speech). The recall of particular aspects of the TRC measure, specifically the recall of visual imagery and speech may be related to left hemisphere activity.

This finding was replicated by Ehrlichman et al. (1985) for the integrated $2 \mathrm{~Hz}$ to $12 \mathrm{~Hz}$ EEG activity obtained within $23 \mathrm{REM}$ and 19 NREM stage 2 sleep intervals. The coefficients computed from the interhemispheric EEG power ratio $(L-R / L+R)$ regressed over three one-minute preawakening time intervals were used as the criterion 
of hemispheric balance. The statistical analyses were conducted using the most extreme left and right dominant regression coefficients paired within each sleep state for each subject. A second analysis was carried out using the mean asymmetry difference score ratio $(L-R / L+R)$ for both the 1 and 3 minute preawakening intervals. Changes in asymmetry were interpreted as reflecting differential hemispheric activation. The results of both analyses indicated no significant difference between left and right predominant REM and NREM sleep awakenings for the total recall count measure. Increased recall of content in terms of the number of words was not associated with differential hemispheric activation for both within REM and within NREM stage 2 sleep. These findings would appear to be in opposition to the theoretical view linking the amount of recall to greater involvement of the left hemisphere. In addition, it was expected that the EEG of those reports associated with more visual content would reflect greater right hemisphere activation, while those showing more verbal content would be related to left hemisphere activity. Contrary to expectations, the visual action scale was significantly related to increasing left hemisphere activity with a similar nonsignificant trend for explicit speech.

For the combined stage 2 and REM EEG postawakening interval, Moffitt et al. (1982) found failure to recall was associated with significantly greater beta power in the right hemisphere while on that measure the EEG showed no interhemispheric differences with respect to successful recall (hemispherically balanced). This finding would appear to support results of waking performance studies by 
Doricchi, Violani \& Milana (1990) and Bertini et al. (1985). These authors reported that good dream recallers showed more balanced activity between hemispheres while low or poor recallers showed greater asymmetries on tasks measuring cognitive functions believed to be involved in dreaming. Thus, successful recall may imply equal contribution of each hemisphere while low or nonrecall may involve greater unilateral (left or right) hemispheric functioning.

In summary, studies employing EEG measures to determine hemispheric activation found little evidence to support differential hemispheric involvement at the preawakening interval with respect to dream recall. Moreover, once established as a successful recall, it appeared that the amount of information reported was not related to laterality EEG measures. However, when the nature of the content (verbal, visual) was taken into account, some significant differences in hemispheric involvement emerged. Those differences, however, were not in the expected direction, since both visual and verbal characteristics were related to greater left rather than right hemisphere activation. In addition to the paucity of research, the general lack of positive results may be attributed to a combination of differing interhemispheric EEG measures, electrode placements and sleep states examined. These studies await replication in order to define more clearly the relationship between the hemispheres and dream recall performance.

1.6. Dream recall and EEG: Further considerations

Overall, the review of the empirical research literature offered only wak support to Koukkou and Lehmann's proposed state 
dependency of recall with EEG-defined activation. However, no strong conclusions may be drawn given the small number of studies available. In addition, comparisons across studies are limited due to the differing statistical analyses used, namely between and within subject analyses, different criteria defining recall, the varying preawakening EEG interval analyzed ranging from 16 seconds to 5 minutes, and the sleep states examined (REM and NREM stage 2).

There are other shortcomings of the research relating EEG to dream recall. Firstly, there is a lack of studies addressing recall out of NREM sleep. The examination of recall out of NREM stage 2 sleep may provide a better distribution of dream recall performance than does REM sleep which clearly favours recall success over recall failure. In addition, stage 2 sleep unlike REM occurs evenly across the night and may allow a better sampling of all major sleep cycles across the night including the first one preceding REM onset.

Secondly, all of the studies except one, that of Moffitt et al. (1982), applied spectral analysis to the EEG data. Spectral analysis restricts the evaluation of the EEG waveforms to one measure, power. Given that period analysis, unlike spectral analysis, provides a power measure in addition to two percent time in frequency measures independent of amplitude (or power), the use of period analysis may offer a more extensive means of relating EEG to recall.

Thirdly, the present studies confined their analysis to the preawakening EEG intervals. Yet, Moffitt et al. (1982) indicated that the postawakening interval may be related to recall performance. Fiss, Klein \& Bokert (1966) argued that the preawakening EEG 
characteristics of a sleep stage may have effects persisting into the postsleep period, and influence the incidence of recall. However, the postsleep interval may constitute an entirely different processing state than the presleep state affecting the retrieval of the dreamed material (Bertini et al., 1985). Indeed, it has been assumed that the postsleep period corresponded to a wakefulness state, but it may be considered more like a transition state from sleep to wakefulness. It is during this transition period that subjects are asked to recall. For example, Simmons, Ogilvie, Segalowitz \& Janicki (1990) found that just prior to behaviorally identified awakenings, the preawakening interval was associated with changes in high frequency EEG activity (power in the alpha, sigma and beta frequency bands) preceding changes in the slower frequencies. Some EEG characteristics typically associated with sleep only, such as delta waves, were found to persist into wakefulness, at least for the period of transition between sleeping and waking. Similarly, Pigeau (1985) reported that the waking EEG of one subjects' awakening from stage 4 sleep retained high levels of low amplitude delta frequency with the appearance of higher frequency activity. The slow wave activity may possibly account for the greater drowsiness typically associated with awakenings from that sleep stage. Whether it is the differential amount of slow wave activity that affects dream recall or whether it is the amount of fast frequency activity that facilitates retrieval of information upon awakening has yet to be examined. As the postsleep interval characterizes the period closest to the retrieval of the sleep experience, it should be more closely examined. 
Similarly, there is no research examining the relationship between the sleeping and waking EEG states in relation to recall and nonrecall. According to Koukkou and Lehmann (1983), dream recall may depend on the similarity between the sleeping and waking EEG states. This similarity may be described in terms of the magnitude of difference between the two states, preawakening and postawakening EEG intervals; the greater the similarity of EEG between the sleeping and waking states, the greater the recall.

This proposal is based on the assumption that recalling dreams involves the ability to transfer information from one state (sleeping) to another (waking) across some sleep-wake boundary or 'warp' (Laughlin, McManus, Rubinstein \& Shearer, 1986). Cohen (1979) argued that the difficulty for a dreamed experience in the preawakening state to be recollected in the following state may depend on the disparity of psychobiological distance between the two states. Similarly, individuals characterized by a less permeable, more distinct boundary (Hartmann, 1984) or by a dissimilar neural organization between siates (Cohen 1979; Laughlin et al., 1986) would more likely show a greater discontinuity of experience in crossing states. This lack of continuity may hinder the transfer of dreamed material and render the recall of dreams more difficult.

Some evidence to support this view is provided by the greater incidence of recall out of REM relative to stage 2 sleep. REM is characterized by a closer to wakefulness EEG state while stage 2 with its less frequent dream recall depicts a greater electrophysiological distance between sleeping and waking states. Additional empirical 
evidence was provided by Goodenough et al. (1965) indicating that an abrupt method of awakening following REM sleep resulted in a high rate of dream recall whereas an abrupt awakening from NREM sleep increased contentless reporting. These findings suggest that the greater magnitude of differences in EEG between NREM sleep and the sudden entry into wakefulness would account for the loss of reportable contents following an abrupt awakening. Conversely, an abrupt awakening from REM sleep, which is electrophysiologically closer to the waking state might allow a quick and efficient crossing into wakefulness with less epportunity for the experience to fade away.

In support of Koukkou and Lehmann's principle, Armitage (1986) found high frequency dreams recallers depicted a greater continuity or integrity of rhythmic cortical activity in the transition from sleep to wakefulness than low recallers. Similarly, Moffitt and Hoffmann (1987) found that high recallers showed a greater number and a more complex patterning of preawakening to postawakening EEG interrelations. In sum, there is evidence to support the notion that the magnitude of change between sleeping and waking states, defined in EEG terms, may differentially influence dream recall.

Fourthly, the assessment of recall performance has usually included two end points of a continuum, success and failure to recall. The intermediate points of this continuum may be described quite arbitrarily depending on the dimensions of interest. One category of recall that may be worth investigating is the contentless recall. During wakefulness, this experience of being unable to recall 
sumething yet knowing that it is known has been substantiated as an empirical phenomenon (Baddeley, 1990). Yet, little information exists about the memory processes involved in the failure to recall the details of the to-be-retrieved content. However, feelings of knowing about unreportable items were found to be directly related to the degree of initial learning (Klatzky, 1984) and suggest that some factors at the encoding level of processing may affect recall performance. In sleep, the contentless dream recall (no recall of content but a clear impression of having dreamed) has been described as a qualitatively different type of failure to recall from the dreamless recall (no feelings of having dreamed and no recall of content) (Cohen, 1972). In addition, Cohen suggested that the contentless recall in comparison to the dreamless recall may correspond to a different psychological experience with possible physiological correlates. One study, that of Meir (1989) supports this view. Meir found that following REM awakenings, no content reports showed intermediate EEG power values between those recorded for successful and dreamless recalls. In agreement with Cohen (1972), the contentless reports were more similar to the failure to recall (in terms of EEG) than the successful recall indicating that such reports characterize a different kind of failure to report rather than a kind of successful recall without the content available. Preliminary analyses using pilot data for this research failed to replicated Meir's (1989) findings for the preawakening interval. Rather a trend emerged differentiating contentless recall from nonrecall at the postawakening interval on measures of laterality between 
hemispheres. Those findings may be considered tentative in view of the small sample size examined and await replication.

Other research linking cortical arousal to contentless reporting indicated such reports occur more often following awakenings from the less activated NREM sleep that REM sleep with its high degree of activation (Baekeland, 1970; Fiss et al., 1966; Goodenough et al., 1965; Moffitt et al., 1982). In addition, Zimmerman (1970) using an auditory threshold measure to reflect the sleeper's proximity to wakefulness state, found that light sleepers reported dreaming out of stage 2 more often than deep sleepers who reported more thoughtlike dreams. These findings suggest that different levels of physiological arousal may be related to a different quality of recall, such as contentless reporting.

Findings by Moffitt et al. (1982) and Schachter (1987) indicated there were individual differences associated with the incidence of contentless recall. Self-reported high dream recallers provided consistently less contentless reports than low dream recallers following REM and stage 2 awakenings. However, Schachter (1987) found that the differences between high and low recallers were greatly reduced for stage 2 sleep. In this paper, subjects were for the majority intermediate dream recallers thus lessening the individual difference variability. The relationship between EEG and recall performance may thus be more clearly delineated within the stage 2 sleep state.

Other factors affecting the rate of contentless reporting from NREM sleep are the method of awakening and distractions upon 
awakening. In stage 2 sleep, there are more contentless reports following abrupt rather than gradual awakenings (Goodenough et al., 1965). Since gradual arousals may promote descriptions of hypnopompic experiences, an examination of stage 2 sleep reporting warrants the use of an abrupt method of awakening. In addition, contentless reports occur more often when attention is not focused on the sleep experience immediately upon awakening (Cohen, 1974). Thus, in this study, the awakenings were performed using an abrupt method. In addition, with the lights off, the subjects were instructed to remain still and keep their eyes closed to allow minimal distractions immediately upon awakening.

Finally, the few studies that have included both males and females as subjects have not investigated EEG differences with gender as a moderator variable affecting dream recall (Meir, 1989; Williamson et al., 1986; Wollman \& Antrobus, 1987). It is well known that there exist gender differences in the content elements of the dream reports, elements which appear to reflect the differential waking concerns of the sexes (Cohen, 1979; Hall, 1984). However, Purcell (1987) found no differences in the dreams of males and females in levels of dream self-reflectiveness or dream control. Using a different analytic approach to Purcell's data, Darling (1989) found that females in comparison to males reported a significantly greater incidence of lucid dreaming (the recall of being aware of dreaming while dreaming) following lucidity induction training. Together, these findings do not constitute strong evidence to suggest a different dream production system or dream self-regulatory 
process in relation to gender.

Gender differences in sleep EEG have also been investigated. Matousek and Petersen (1973) reported a higher amount of beta frequency activity in females than in males. More recent findings by Dijk, Beersma, Bloem (1989) indicated that in REM and NREM sleep (stages 2, 3, and 4), females showed consistently higher power densities in all frequencies analyzed (delta to alpha range) especially delta, than males. These differences persisted across all sleep cycles throughout the night. The differences appeared not to be associated with different sleep regulatory processes as evidenced following recovery from sleep deprivation. Rather differences could be attributed to anatomical differences such as skull thickness and a generally smaller head size in the females but may more. likely be due to differing neural mechanisms. More specifically, Flor-Henry, Koles \& Reddon (1984) suggested that relative to men, the thicker cortical gray mantle and faster rate of cerebral blood flow of women may be responsible for their greater cortical EEG power and hemispheric coherence.

In terms of interhemispheric activity, it has generally been found that females are more synchronized (greater interhemispheric coherence) and show less lateralization of functions than males in waking performance (Flor-Henry et al., 1984; Springer \& Deutsch, 1989). However, in a comprehensive review of the EEG asymmetry and performance literature up to 1983, Pigeau (1983) found contradictory evidence suggesting females were more, less and equally lateralized relative to males on the various tasks performed. 
During sleep, Reinsel, Antrobus \& Fein (1989) found no significant hemispheric EEG power differences between males and females for central and tempoparietal electrode placements. In the transition from sleep to wakefulness, Armitage (1986) found gender differences in interhemispheric EEG activity with females, especially self-reported high dream recallers, maintaining a greater continuity of rhythms in cortical activity between the two states. Preliminary analyses using pilot data for this research indicated some significant interactions of gender with recall. In this paper, EEG differences will be examined with gender as a moderator variable to assess its effect on dream recall performance. 


\section{CHAPTER TWO}

This research proposed examining the relationship between cortical activation as defined by EEG measures computed by period analysis and dream recall. The differences in recall performance will be evaluated within a given state, stage 2 sleep specifically. The purpose was to investigate the state-dependent relationship of recall performance with EEG-defined activation following Koukkou and Lehmann's model. This study included four predefined recall groups: success, contentless, delayed and failure, with cortical activation level indexed by EEG measures. The differences between recall groups in relation to electrophysiological changes will be examined for the 5 minute preawakening, 30 second postawakening, and preawakening to postawakening time intervals. The general research question may be formulated this way: According to Koukkou and Lehmann's proposal of state-dependency of retrieval, does recall performance vary as a function of EEG-defined changes in cortical activity? Principally, can recall groups divided into success and failure, be differentiated using tonic EEG measures computed for the preawakening, postawakening and preawakening to postawakening time intervals?

The cortical activation mctels using EEG as their measure assume that with increasing level. of cognitive activity there is a corresponding increase in cortical arousal. Typically, cortical activation is associated with a reduction in EEG total power, a decrease in low frequency EEG activity (delta power, theta power, or both) or an increase in high frequency EEG activity, especially beta 
power. Using EEG period analytic procedures, high and low frequency EEG activity may also be evidenced in the percentage amount of time per EEG epoch spent in a given frequency category as indexed by zero cross and first derivative events. Generally, the greater the amount of time spent in higher frequency EEG activity the greater the activation level, whereas the greater the amount of time spent in low frequency EEG activity the lower the level of activation. High frequency activity may often be superimposed on low frequency activity and not be detected if only power and zerocross events are examined. The first derivative analysis allows the detection of these high frequencies to be distinguished from the low frequency activity. These patterns of EEG variations defining activation levels are interpreted the same way for the interhemispheric measures. The asymmetry measures also specify which hemisphere depicts the greatest level of EEG activity. Keeping these notions in mind, some hypotheses may be formulated relating dream recall performance to EEG activation levels:

\subsection{Hypotheses}

1a. Based on Koukkou and Lehmann's state-dependency principle of recall, it is expected that dream recall performance increases with increasing levels of EEG-defined cortical activation. Therefore, it is hypothesized that for the preawakening interval the recall groups will be significantly different. Specifically, the successful recall group will exhibit higher levels of activation than the failure to recall group. It is expected that the contentless and delayed recall groups, both showing diminished dream recall performance, should exhibit 
intermediate levels of activation between the successful and failure to recall groups. Particular attention will be paid to changes in total EEG power, as well as delta, theta and beta frequency activity measures which have received the most support in the empirical research literature.

1b. Based on the assumption that cortical activation levels may influence recall at the preawakening level it may be tentatively expected that this relationship extends to the postawakening interval. Therefore, following Koukkou and Lehmann's principle, it is expected that as for the preawakening interval, increasing cortical activation levels will be associated with increasing recall performance at the postawakening interval.

2. Following Koukkou and Lehmann's assumption that the greater the magnitude of differences between the preawakening and corresponding postawakening EEG measures, the more impaired the dream recall, it is expected that the successful recall group would exhibit smaller differences in preawakening to postawakening difference score EEG measures than the failure to recall group. The contentless group and delayed recall groups should show intermediate values.

3a. The most recent theoretical literature suggests that there should be greater involvement of the left hemisphere with increased recall at the preawakening interval. However, empirical data are lacking within stage 2 to support this notion and would appear not to be adequate with regards REM sleep (Lehmann et al., 1981). Thus, at the preawakening interval, it is hypothesized that the successful 
recall group will not differ from the failure to recall group on hemispheric laterality measures.

3b. At the postawakening interval, the theoretical proposals of Koukkou and Lehmann (1983) have not been examined. It is expected that successful recall will be associated with higher activation levels in the left hemisphere than nonrecall. Failure to recall will show right hemisphere activation (Moffitt et al., 1982) or be associated with a lesser involvement of the left hemisphere relative to recall (Antrobus, 1987).

3c. No data exist relating contentless or delayed recall to laterality measures. However, if delayed successes reflect an experience similar to successes, and contentless experiences are similar to failures, than tentatively, it is hypothesized that at the postawakening interval, delayed recall will be left hemisphere activated and contentless recall will show a pattern of hemispheric activation similar to nonrecall.

4. Gender effect: The lack of data relating dream recall with gender precludes the formulation of specific hypotheses. The analyses including gender as a variable are exploratory in nature and will be limited to differences between the success and failure recall groups. 2.2. Method

\subsubsection{Subjects}

Forty undergraduate student volunteers were given course credits for their participation in this experiment (see Appendix A). Twenty subjects were first run as part of a pilot study. The subsequent twenty subjects were then added in order to provide a 
larger sample and increase the statistical power of the analyses. The two groups were run following the same experimental procedures. Of the 40 subjects, there was an equal number of males and females varying in age between 18 and 34 years; mean age 20.0 years (males $=20.7$ years; females $=19.3$ years). Subjects were for the majority $(\mathrm{n}=33)$ intermediate self-reported home dream recallers (betwees one to five dreams a week). Two subjects were infrequent recallers (about once a month) and five were high frequency recallers (every morning). The selection of subjects in those categories was made in order to ensure an adequate sampling of both recall successes and failures. Subjects were functionally righthanded and free of any known medical or sleep disorders. They were asked to refrain from alcohol, caffeine, or drug consumption, as well as atypical napping on the day of the study so as not to disrupt their regular sleep patterns (see Appendix B).

\subsubsection{Apparatus}

The equipment consisted of six Grass P511J preamplifiers with power supply, and a Tektronix dual beam oscilloscope. The EEG signals were digitized at 128 samples per second through 12 bit analog to digital (A/D) converters, stored on hard disc on a Packard Bell computer and copied onto optical laser disks for permanent storage. For the purpose of on-line visual stage identification an Apple Macintosh IIci computer sampled the EEG signals at $60 \mathrm{~Hz}$ and displayed the EEG patterns on a colour monitor. A Sanyo audio cassette recorder was used to record all verbal reports. An intercom system was used for communication with the subject during the 
night.

\subsubsection{Procedure}

Subjects who met selection criteria (see Appendix B) and who agreed to participate, were instructed to arrive at the laboratory one hour before the scheduled 11:00 p.m. bedtime. Upon arrival, they were given a tour of the laboratory for acclimatization purposes. Subjects were then familiarized with the experimental procedures prior to participation in the study (see Appendix C). They were told that there was equal interest in recall and no recall and that they should feel no pressure in having something to report. A consent form (see Appendix D) was then signed by each subject. Electrodes were attached and subjects put to bed.

Four Beckman monopolar silver silver chloride (Ag/Agcl) scalp electrodes with electrolytic gel were used for recording at central (C3, C4) and parietal (P3, P4) sites and referenced to linked ear sites (A1, A2). This report will consider data from the parietal sites only. The parietal location was used because it is associated in wakefulness with the integration and analysis of sensory information and with short term visual memory and retrieval (Kolb \& Winshaw, 1980). In sleep, it has been shown to be a critical region of the cortex involved in dream recall and dreaming, particularly relevant to the production of dream imaging (Gabel, 1988; Greenberg \& Farah, 1986; Murri et al., 1989). In addition, cerebral asymmetries are most likely to predominate at the parietal site (Kolb \& Whinshaw, 1980; Reinsel et al., 1989). The electrodes were secured using collodion. A pair of submental electrodes recorded the EMG. One 
electrode was placed at the supraorbital ridge of the right eye and another was placed at the infraorbital ridge of the left eye to record horizontal and vertical eye movements simultaneously. A ground electrode was placed on the forehead.

A gain of 10,000 X was used for EEG and EOG, with filter settings at $0.3 \mathrm{~Hz}$ and $100 \mathrm{~Hz}$. The EMG amplifier was set to produce a voltage excursion of $2 \mathrm{~V}$ peak to peak at the start of the night. Prior to the experimental sessions the EEG amplifiers were calibrated and counterbalanced left to right between subjects. All electrophysiological signals were recorded simultaneously on hard disc on a Packard Bell computer. The impedance was $<5 \mathrm{~K}$ ohms for EEG electrodes and available for the last 20 subjects only.

After the subjects were prepared for recording, all signals were examined on Tektronix dual beam oscilloscope to ensure that the channels were recording properly. The EEG signals were displayed on a monitor screen for on-line sleep stage identification during the night according to the criteria of Rechtschaffen and Kales (1968). All verbal reports were recorded on audio tape cassettes.

\subsubsection{Experimental awakenings}

Each subject spent one night $(8 \mathrm{hrs})$ sleeping in an electrically shielded sleep chamber. Each subject was awakened by hearing his or her name called firmly on the intercom. The name was called until the experimenter was satisfied the subject was awake as indicated by his response that, yes he was awake, and with the appearance of some body movements and changes in the EEG. All interviews were conducted by the same experimenter following a 
standardized format (see Appendix E) and the verbal reports were recorded on tape cassettes for later transcription. The instructions were read to the subjects each time they were awakened to prevent confusion or misinterpretation of the experimental procedure. With the lights off, subjects were instructed to keep their eyes closed, to remain as still as possible and to describe with as much detail as possible any experiences they were having before being called. These instructions were important in reducing body movement and distraction at the time of awakening as these may interfere with recall (Cohen, 1974). Following a verbal report subjects were repeatedly asked if they had anything else to report until they had nothing left to report. When they were not sure anything occurred or if they reported no recall, they were then asked to think for a moment and see if anything came back to them. In order to prevent subjects from going back to sleep, they were only allowed up to 15 seconds to respond, after which subjects were again asked for a response. Following the verbal reports subjects were asked to respond to four probes and a question characterizing their subjective sleep experience prior to awakening (see Appendix E). In the morning they were asked to respond to questionnaire items (Fenigstein, Scheier \& Buss, 1975) assessing social desirability and ability to focus on inner experiences (see Appendix F). The results for these subjective dimensions will not be examined in this paper. Subjects were awakened a maximum of four times across the night. All awakenings were made following an accumulated five minutes into stage 2 sleep within each successive NREM-REM sleep 
cycles. The first awakening was made prior to REM onset but following the accumulation of at least 10 minutes of stage 4 sleep to preclude the disturbance of the onset of sleep. The remaining awakenings were made at least 30 minutes after the termination of a REM period and after five minutes of a continuous accumulation of stage 2 sleep in order to preclude any effects of REM physiology in recall performance. The awakenings lasted on average 3 to 4 minutes each.

\subsubsection{Classification of reports}

The reports collected were transcribed and classified by raters into four predefined categories of recall: successful, contentless, delayed and failure (See Appendix G). Each of these categories was defined as follows: 1) Successful recall: Any report of some content that could be described and attributed by subjects to the preawakening period. Reports could be of dreaming, thinking or feeling. The content of the reports may be a visual image, an experience in another sensory modality (with or without visual imagery) or an emotional experience (affect). A successful recall was classified in either the delayed (see delayed recall) or clear success category. The clear success corresponded to any reports of some content that could be described by subjects immediately upon awakening. 2) Contentless recall: Subjects claimed to have been dreaming or that something was going through their mind before being called but they could not say or describe what it was. No content was available; there was an inability to recollect any details of the sleep experience. 3) Delayed recall: Included the delayed 
contentless and delayed successful reports. In both cases, subjects first claimed nothing was happening or at least that they were not sure. When asked to think about it, subjects claimed something was happening or reported the content. 4) Failure to recall: After being given the opportunity to think about it, subjects were still unable to report anything at all. They didn't think anything was happening; their mind was blank; they had no impression of dreaming.

\subsubsection{EEG quantification procedures}

Stage scores were assigned to the electrophysiological signals using a computer algorithm (Hoffmann et al., 1984). The stage scores of the 35 minute period of sleep preceding an awakening and the 30 seconds following an awakening were then verified using visual stage scoring procedures following Rechtschaffen and Kales (1968) criteria. All electrophysiological records contaminated with muscle and morement artifacts were eliminated from analysis. Digital period analyses described in Hoffmann, Moffitt, Shearer, Sussman \& Wells (1979) were used to evaluate the EEG signals of each 30 second epoch of the 5 minute preawakening and 30 second postawakening sleep intervals, for each lead. The measures provided by the two EEG channels were then averaged over both hemispheres.

These analyses yielded three measures: two measures of percent time in frequency per epoch (half wave zero-cross and full wave first derivative) and one full wave power measure for each of five frequency bands. An average total power measure was also computed. The frequency bands use.t were: delta $(0.5$ to $<4.0 \mathrm{~Hz})$, theta $(4.0$ to $<8.0 \mathrm{~Hz})$, alpha $(8.0$ to $<12.0 \mathrm{~Hz})$, sigma $(12.0$ to $<16.0$ 
$\mathrm{Hz}$ ) and beta $(16.0$ to $30 \mathrm{~Hz})$. EOG analyses counted the number of eye movement excursions above $50 \mu \mathrm{V}$ and the integrated amplitude of all eye movements. The EMG analysis provided a value for the integrated EMG activity during the epoch. In addition, for each of the periud analysis measures, a relative hemispheric asymmetry measure expressed as the difference ratio $(L-R) /(L+R) \times 100$ was calculated. A positive ratio reflects greater left hemisphere activity, while a negative ratio reflects greater right hemisphere activity. Finally, difference scores between corresponding preawakening and postawakening averaged EEG measures, excluding hemispheric asymmetry measures, were computed.

The use of simple difference scores to measure change has been criticized mainly because of their necessary dependence on prescore values and their low reliability (Lord, 1963; Cronbach \& Furby, 1970). A difference score is defined as the difference between a postscore and a prescore. The effect of the subtraction is to remove the initial effect of the prescore from the final postscore level in order to produce a measure that would reflect change between two points in time. However, the difference score does not reflect true change but rather its magnitude varies as a function of the strength of the dependency between the change score and the initial value. This relationship holds unless the correlation between prelevel and change score value equals zero (Cohen \& Cohen, 1983). According to the law of initial value (Benjamin, 1963), it is usually assumed that the change score and prelevel score are negatively related (Linn \& Slinde, 1977). When this occurs, it is expected that 
large change values will be observed for subjects with low initial values whereas subjects with high initial scores would rarely show large change values. However, it is possible that change scores and initial level be positively correlated (Zimmerman \& Williams, 1982). A positive correlation results when subjects who score highly initially show large gains. In either case, change scores are largely dependent on the prelevel scores.

Various techniques such as the use of residualized difference scores have been proposed to compensate for the effect of initial values (Benjamin, 1963; Linn \& Slinde, 1977; Lord, 1963). A residualized difference score is computed by subtracting the predicted postscore, $Y^{\prime}$, from the corresponding observed postscore, $Y$. The predicted score corresponds to the linear regression of $Y$ on the preawakening $X$ score. The part of the postscore information that is linearly predicted from the prescore is removed. Unlike simple difference scores, residualized differences have a zero correlation with the preawakening scores.

Thus, when using simple difference scores to measure change, residualized scores may be used to verify that the change recorded is not a function of prelevel differences between individuals. If both techniques produce the same results for a given analysis, then the simple difference scores are considered meaningful indicators of change and are interpreted as such. In this paper, simple difference scores were corrected for any dependencies on initial preawakening level by computing a residualized difference score for each EEG parameter and results of the two techniques compared. 
Several investigators have demonstrated that difference scores have low reliability (Cronbach \& Furby, 1970; Linn \& Slinde, 1977; Lord, 1963). However, recent research by Rogosa and Willett (1983), Williams and Zimmerman (1977), Zimmerman and Williams (1982) indicated that under certain assumptions regarding the reliability coeffficients and standard deviations of prescores and postscores, the reliability of change scores could be high. More specifically, Sharma and Gupta (1986) demonstrated the importance of the correlation between the initial and final scores and of the size of the ratio of their standard deviations in determining the reliability of the difference scores.

More importantly, the reliability of the difference score depends on the reliability of the instrument used to obtain the presccre and postscore data. Generally, the use of EEG recording techniques have been shown to produce reliable data (Niedermeyer \& Lopes Da Silva, 1982). In addition, the distribution of each EEG variable set was examined for floor and ceiling effects and all outlying cases were removed. Further, the statistics of the observed prescores and postscores were inspected, principally their correlations and ratios of standard deviations. Although the use of difference scores in some experimental situations may be said to be unreliable, it is believed that the use of EEG difference scores can be an accurate and meaningful measure of change.

\subsubsection{Statistical analyses (clear success vs failure)}

Separate unpaired t-tests (two-tailed) were used to assess the differences between clear success and failure to recall groups on each 
EEG sleep parameter. Separate analyses of variance (ANOVAs) were used to differentiate the four recall groups: clear success, delayed success, contentless and failure. There was an insufficient number of observations in the delayed contentless group to examine it as a separate category of recall. Taking into consideration the increase in Type I error due to the possible correlations between observations taken from the same subject (Zimmerman, Williams \& Zumbo, in press) and the large number of independent statistical tests performed, group differences were considered significant only when the alpha probability was equal to or less than the .025 level. In addition, separate two-way ANOVAs were used to assess the differences between recall groups (clear success vs failure) with gender as a factor.

\subsection{Results}

\subsubsection{Dream Recall: Descriptives}

Interrater agreement followed an exact agreement procedure of 2 out of 3 judges on the first independent round. Four ambiguous cases could not be rated and were eliminated from analysis. For the remaining 126 cases available, the Pearson's correlation coefficient between any two judges indicated high interrater reliabilities i.e., .91, .92 and .97 .

A total of 126 awakenings were used resulting in $39.7 \%(\underline{n}=50)$ successes, $10.3 \%(\underline{n}=13)$ contentless, $19.8 \%(\underline{n}=25)$ delayed and $30.2 \%$ $(n=38)$ failures. The delayed group included 18 delayed successes and 7 delayed contentless reports. When the delayed successful reports were lumped together with the successful reports, and all 
other reports were combined, the distribution of recall across the night was $54 \%$ successes and $46 \%$ failures.

\subsubsection{Dream Recall and wakeup ordinal position}

Across all recall groups, the number of awakenings was fairly consistent for the first three wakeups (37, 40 and 34 , respectively) with only 15 awakenings in the fourth wakeup position. As shown in Figure 1, the number of clear successes increased from the first to the second wakeup with a slight decrease in the third wakeup. The number of failures to recall decreased from the first to the second wakeup and increased slightly in the third wakeup. For both groups, the greatest change in recall occurred between the first and second wakeup. There was an approximately equal number of clear successes and failures following the first wakeup (10 successes vs 14 failures) while there were more than twice as many successes as failures following the second awakening (19 successes vs 8 failures). There was approximately the same number of recall successes and failures for the third (15 successes, 11 failures) and fourth wakeups (6 successes, 5 failures) (See Figure 1). The majority of contentless reports were found following the first awakening. The delayed successful reports were evenly distributed across the first three wakeups while there were clearly more delayed contentless reports following the second awakening. If clear successes are combined with delayed successes and compared to all other reports, the distribution of recall across the night follows the patterns typically reported (See Figure 2). There are more failures than successes following the first awakening with a reversed trend towards 


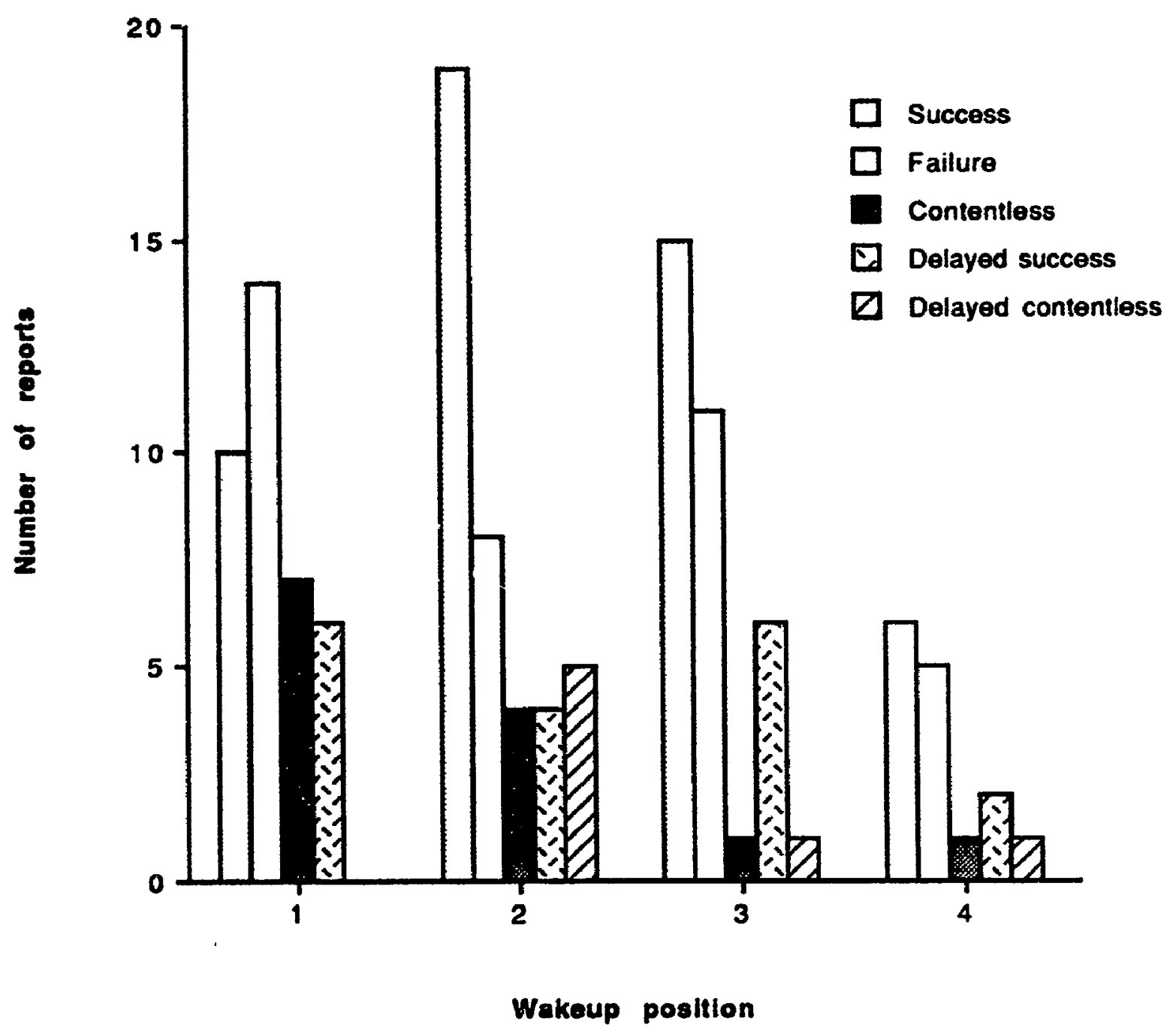

Figure 1. Distribution of dream reports within recall groups across successive wakeups. 


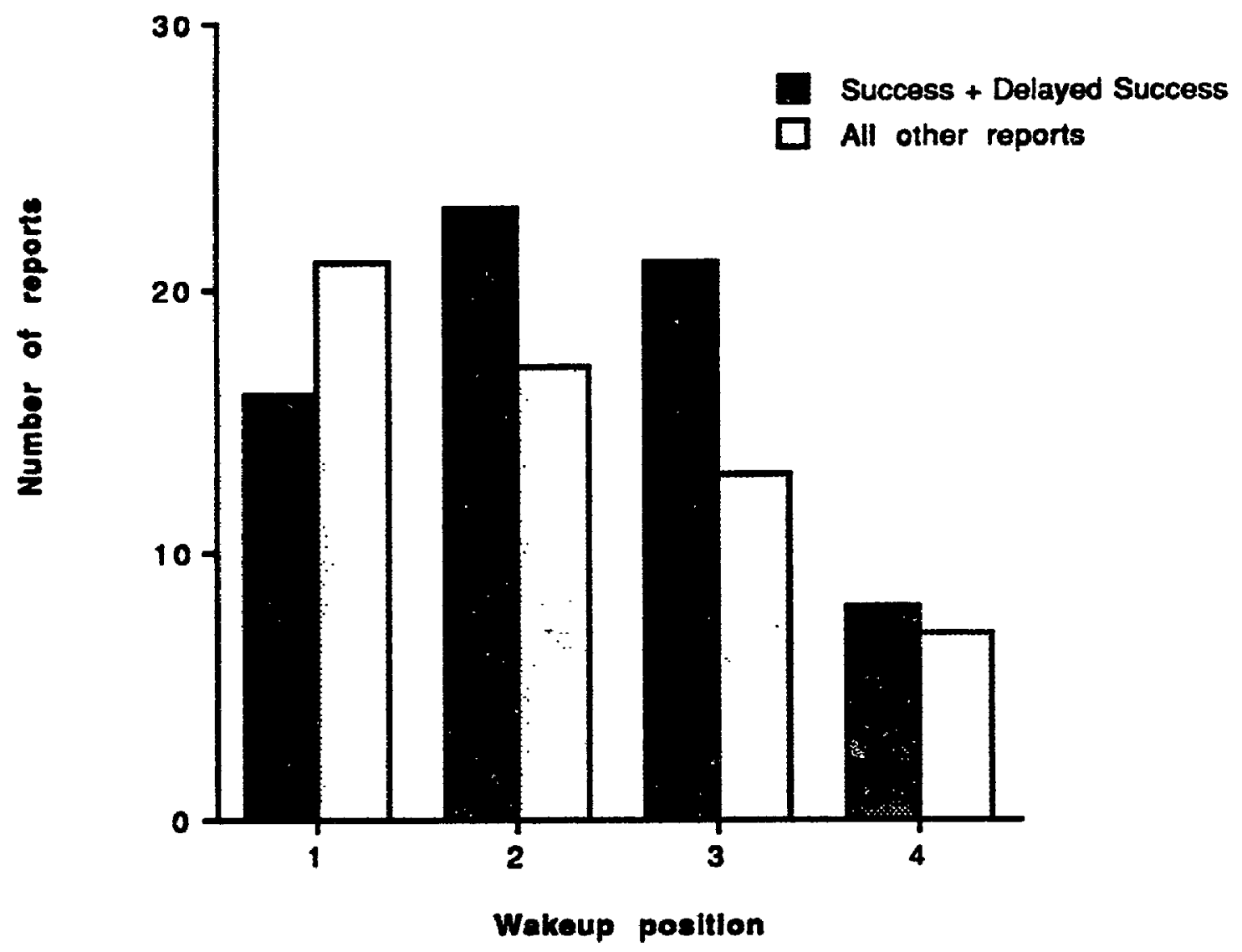

Figure 2. Distribution of dream reports within recall (success and delayed success) and nonrecall groups (contentless, delayed contentless and failure) across successive wakeup. 
increased recall in the second wakeup position relative to nonrecall.

Since there was an approximately equal number of clear successes and failures following the first awakening (10 successes and 14 failures) while there were almost twice as many successes as failures (40 vs 24) for the last three awakenings combined, a posthoc two factor ANOVA (recall by wakeup) was performed on each preawakening EEG sleep measure. These analyses assessed whether the greater recall in the first three awakenings could result from any preceding REM effects and to assess the EEG effects of wakeup position on recall. The analyses excluded the fourth wakeups as there was an insufficient number of reports for that awakening position. Results indicated no significant interactions of recall by wakeup position on any EEG parameter. There were significant main effects of wakeup on EOG $(E(2,71)=8.53, \mathfrak{p}=.0005)$, EOG power $(E(2,71)=16.52, p=.0001)$, theta power $(E(2,71)=8.53, p=.0005)$, and total power $(E(2,71)=6.39, \mathfrak{p}=.0028)$. For each of these variables, the means showed a decreasing trend across the night. 2.3.3. Gender and dream recall: Descriptives Of a total 88 clear successes and failures, there were 46 reports from males and 42 from females. The distribution of recall - successes and failures between males and females was fairly equal. Of 50 successes, 16 males contributed 28 reports and 13 females provided 22 reports. Of 38 failures, there were 18 reports from 12 males and 20 reports from 10 females. The mean number of successes per subject was 1.7 for both males and females. The mean number of failures per female subject was 2 and 1.5 for males. 
2.3.4. Preawakening EEG and dream recall: Clear success vs failure

In all, 38 subjects contributed a total of 88 reports. There were 50 clear successes and 38 failures. For the preawakening interval, the unpaired t-tests on each of 35 EEG parameters revealed no statistically significant differences between recall and nonrecall. The integrated muscle activity (EMG) variable approached significance with $t(85)=2.23, \mathfrak{R}=.028$. On that variable the means indicated a trend toward greater muscle activity for nonrecall than recall. The overall activation measure indexed as the average total power failed to reach significance $(\mathrm{R}=.054)$. However, the means were in the expected direction with greater power prior to nonrecall than recall (See Table 1).

\subsubsection{Postawakening EEG and dream recall: Clear success vs failure}

For the postawakening interval, unpaired t-tests on each of 35 EEG parameters indicated a significant difference for the EMG measure, $\mathrm{t}(85)=3.123, \mathrm{R}=.002$ and for the sigma first derivative measure, $\mathrm{t}(85)=2.928, \mathfrak{R}=.004$. As depicted in Table 1 , there was greater muscle activity recorded for recall relative to nonrecall. However, there was greater sigma activity prior to failure to recall than successful recall.

\subsubsection{Preawakening to postawakening EEG differences and} dream recall: Clear success vs failure

Unpaired L-tests applied to each of 19 difference scores between preawakening and postawakening intervals revealed a significantly greater change on the EMG and sigma first derivative measures $\mathrm{t}(85)=3.39, \mathrm{p}=.001$ and $\mathrm{t}(86)=2.35, \mathrm{p}=.02$, 
Table 1. Recall group differences on electrophysiological (EEG) measures.

\begin{tabular}{|c|c|c|c|c|}
\hline \multirow[b]{2}{*}{ Preawakening EEG } & & \multicolumn{2}{|c|}{ Recall categories } & \multirow[b]{2}{*}{ P value } \\
\hline & & Success & Eailure & \\
\hline EMG & $\stackrel{\mathbf{M}}{\mathbf{S D}}$ & $\begin{array}{r}1867.88 \\
531.20\end{array}$ & $\begin{array}{r}2208.18 \\
882.43\end{array}$ & $p=.028$ \\
\hline Total power & $\frac{\mathbf{M}}{\mathbf{S D}}$ & $\begin{array}{l}708.00 \\
122.53\end{array}$ & $\begin{array}{l}761.79 \\
134.27\end{array}$ & $p=.054$ \\
\hline Postawakening EEG & & Success & Eailure & P value \\
\hline EMG & $\frac{\mathbf{M}}{\mathrm{SD}}$ & $\begin{array}{r}12247.68 \\
3741.40\end{array}$ & $\begin{array}{l}9958.86 \\
2812.96\end{array}$ & $p=.002$ \\
\hline $\begin{array}{l}\text { Sigma first } \\
\text { derivative }\end{array}$ & $\frac{\mathbf{M}}{\mathrm{SD}}$ & $\begin{array}{l}9.12 \\
2.97\end{array}$ & $\begin{array}{r}11.34 \\
4.10\end{array}$ & $p=.004$ \\
\hline $\begin{array}{l}\text { Preawakening to } \\
\text { postawakening EEG }\end{array}$ & & Success & Eailure & P value \\
\hline EMG & $\frac{\mathbf{M}}{\mathrm{SD}}$ & $\begin{array}{r}10319.52 \\
3918.77\end{array}$ & $\begin{array}{l}7727.65 \\
2902.79\end{array}$ & $p=.001$ \\
\hline $\begin{array}{l}\text { Sigma first } \\
\text { derivative }\end{array}$ & $\stackrel{\mathbf{M}}{\mathbf{S D}}$ & $\begin{array}{l}8.26 \\
3.95\end{array}$ & $\begin{array}{l}6.26 \\
3.94\end{array}$ & $p=.020$ \\
\hline
\end{tabular}

Note. The units of analysis for the EEG measures are arbitrary and arise from the algorithm used in the $A / D$ converters. 
respectively). In both cases there was a greater change preawakening to postawakening associated with recall than nonrecall (see Table 1).

Separate unpaired t-test analyses using residualized difference scores produced the same significant findings as those reported using simple difference scores. There was a significant difference for the EMG measure $t(86)=2.344, R=.021$ and for the sigma first derivative ineasure $\mathrm{t}(86)=2.656, \mathrm{R}=.009$.

Separate nenparametric Mann-Whitney U-tests were performed on each EEG parameter in each preawakening, postawakening and preawakening to postawakening data set. These analyses produced the same results as those reported for the parametric analyses.

2.3.7. Within subject analysis: Clear success vs failure

Since only 13 subjects contributed both a recall and a nonrecall, and because this number was not representative of the total sample examined, within subject comparisons to control for individual EEG differences were not performed.

\subsubsection{Gender and dream recall}

There was a significant main effect of gender for the asymmetry measure of beta first derivative $(E(1,84)=5.572$, $\mathrm{p}=.021$ ). The means indicated the males were balanced on that measure while the females had slightly more beta first derivative activity in the right hemisphere $(\underline{M}$ for males $=-0.043 ; \underline{M}$ for females $=-1.86$ ). In addition, at the $\mathrm{R}<.05$ level, there was a significant main effect of gender on the sigma first derivative measure $(E(1,84)=$ 
4.69, $\mathrm{R}=.033$ ). The means indicated a trend for a slightly greater amount of sigma first derivative activity for females than males $(\underline{M}$ for females $=18.024 ; \underline{M}$ for males $=17.087)$. The two-factor ANOVAs indicated no significant interaction between recall and gender at the $\mathrm{R}<.025$ level. However, at the more liberal $\mathrm{R}<.05$ level, there was a significant interaction of recall by gender on the theta power measure $(E(1,84)=4.57, p=.035)$. Tests of simple main effects indicated that females had a significantly greater amount of theta power than males in association with successful recall $(E(1,84)=8.87, \mathrm{p}<.01)$ (See Figure 3).

For the postawakening interval the two-factor ANOVAs indicated a significant recall by gender interaction for the interhemispheric measure of alpha power $(E(1,84)=5.91, p=.017)$. Tests of simple main effects revealed a significant recall difference within the female group, on that measure. Successful recall was. associated with alpha power activity in the left hemisphere, whireas failure to recall was related to alpha power activity in the right hemisphere $(\mathbb{E}(1,84)=7.95, \mathrm{p}<.01)$. In addition, the tests of simple main effects revealed a significant gender difference in association with nonrecall. Males showed alpha power activity in the left hemisphere, whereas females showed alpha power activity in the right hemisphere $(E(1,84)=4.83, p<.05)$ (See Figure 4).

Two-way ANOVAs applied to difference scores at the preawakening to postawakening interval failed to produce any significant gender by recall interactions or main effect. 
73

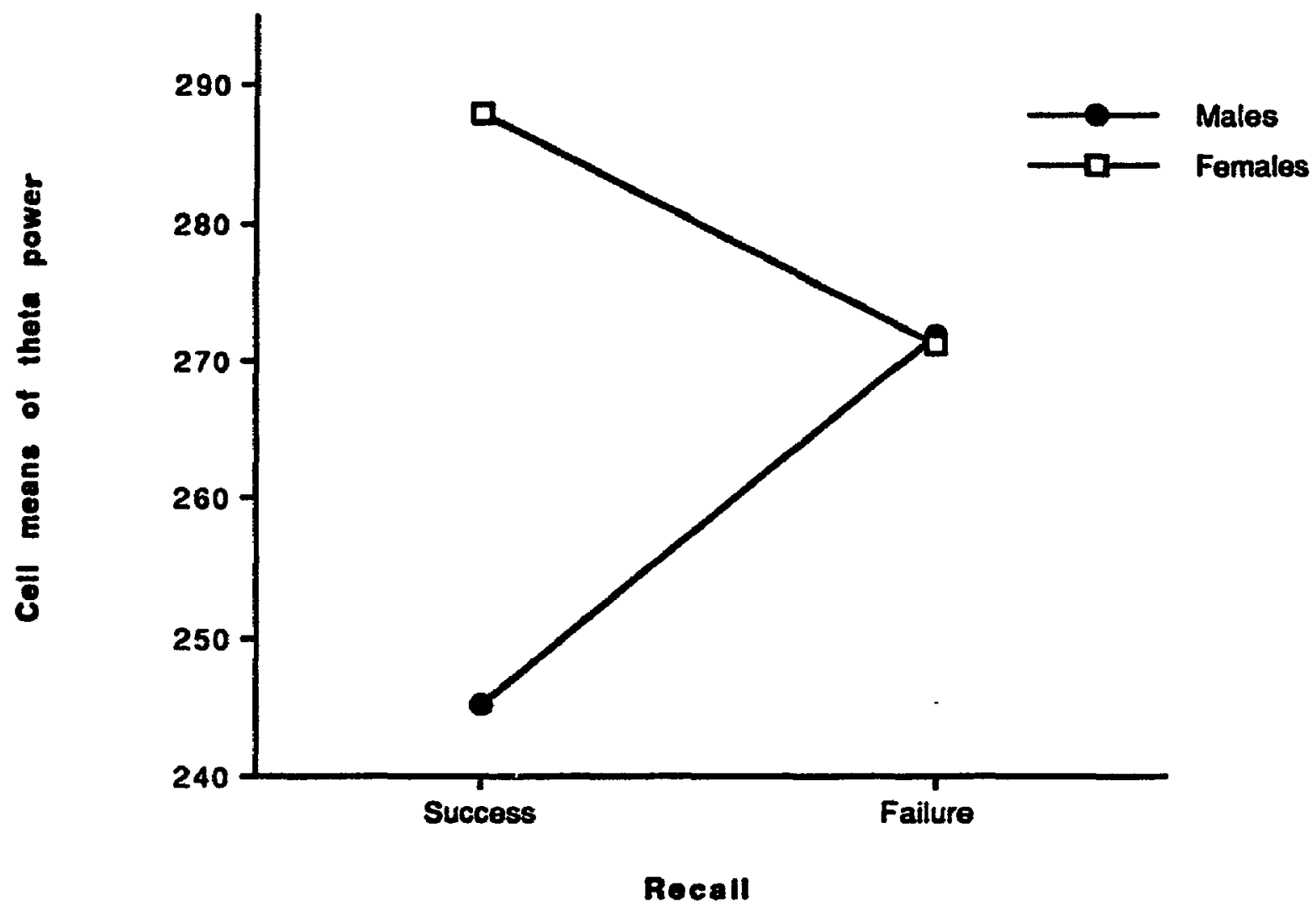

Figure 3. Means indicating significant interaction between gender and recall on preawakening EEG theta power measure. The units of analysis for Theta power are arbitrary and arise from the algorithm used in the A/D converters. 


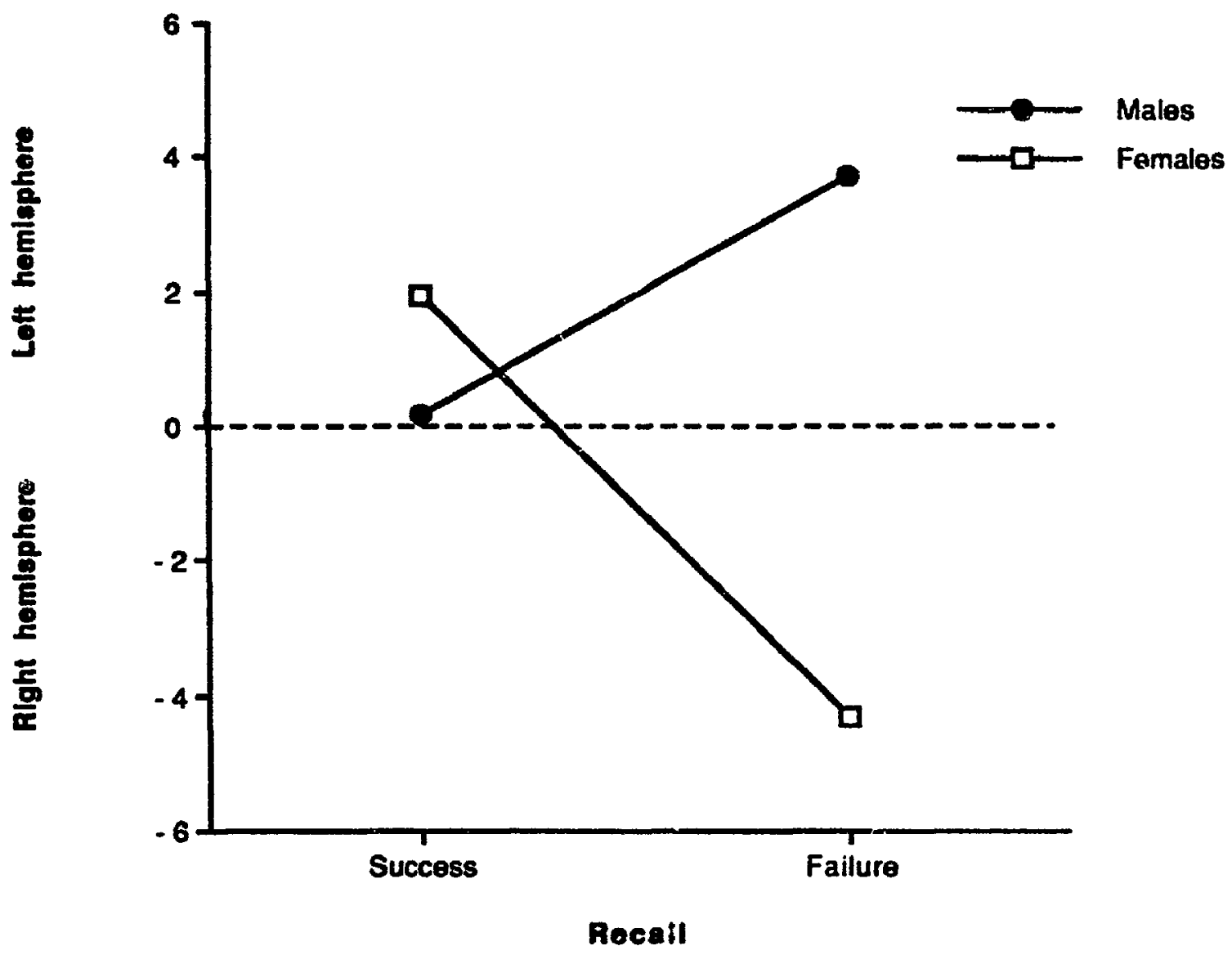

Figure 4. Means indicating significant interaction between gender and recall on postawakening alpha power EEG asymmetry measure. 
2.3.9. Multivariate analyses: Clear success vs failure

In order to determine if a linear combination of EEG variables might be a more powerful predictor of recall than EEG variables considered individually, it was intended that a separate stepwise regression analysis be performed on selected preawakening, postawakening and preawakening to postawakening EEG parameters. Principal components analysis applied on each respective EEG data set would be used to reduce the large number of variables to a smaller set of predictors for regression analysis. Variables loading highly (an absolute loading equal to or greater than .30) on the same component as the recall variable (success and failure groups combined) would be selected as useful predictors in the regression equations.

To optimize the reliability of the components analysis, certain criteria had to be met. First, the adequacy of the data wit was assessed through the inspection of univariate and multivariate outliers; all outlying cases were removed from subsequent analyses. In addition, variables that were singular (a composite of other varialles) or dichotomous, with very uneven splits (i.e. $80 \%-20 \%$ ) between the two categories were not included in the analyses (Tabachnick \& Fiddell, 1989). Secondly, a preliminary components analysis was performed to estimate the factorability of the correlation matrix and to estimate the efficiency of sampling of the variable set. The matrix should include a large number of correlations with coefficient values equal to or greater than .30 . If all correlations are low, it is unlikely that the variables share 
common component structures. An inspection of the zero-order correlation matrices of the preawakening, postawakening and difference scores EEG data sets revealed that overall each matrix was appropriate for components analysis comprising a sufficient number of high correlations. However, in each of the three matrices, the recall variable had no high correlations with any other variables in the set. Similarly, the squared multiple correlation (SMC) of a variable with all other variables taken as a linear combination should be high (minimum absolute value of .40 ), as it represents that variable's proportion of variance accounted for by the other variables in the matrix. For recall, the squared multiple correlations at the preawakening and postawakening intervals barely met the minimum requirement with values of 0.44 and 0.42 respectively, while the value at the preawakening to postawakening interval was below acceptable levels $\left(\mathrm{SN}^{-}=.245\right)$. A Kaiser-Meyer-Olkin measure of overall sampling adequacy with a value of .6 is required for a good analysis, with individual variables with values equal to or less than .5 excluded from analysis. The overall measure of sampling adequacy is an index of the extent to which a matrix conforms to the requirements of near zero parıal correlations and large multiple correlations between variables. In the same manner, the individual variable's measure of sampling adequacy involves then: correlations relating to a particular variable only. The partial correlations are the correlations that exist between two variables with the linear effects of all other variables partialled out. Findings indicated that the overall sampling efficiency of the three data sets was adequate. 
However, in each data set, the recall variable had an efficiency measure below acceptable levels for analysis.

Taken together, these evaluations indicated that there was sufficient variability in each data set to justify performing a principal components analysis. However, on the basis of low zero-order correlations, low squared multiple correlations and a low individual sampling efficiency index, the recall variable would account for little variance in the overall matrix or conversely, the matrix of EEG variables would contribute little to the predictability of recall. Therefore, the components analyses were discontinued.

2.3.10. Other analyses

A grouping made up of clear success, delayed success, contentless and failure groups was used to examine whether the delayed success and contentless reports varied relative to the success and failure categories. Due to the small number of cases in the delayed success $(\underline{n}=18)$ and contentless $(\underline{n}=13)$ categories, a random sample of 18 observations was taken within the clear success and failure groups to provide a more adequate test of differences between groups. There was an insufficient number of delayed contentiess reports $(\underline{n}=7)$ to include these cases as a separate group in the analysis.

For the preawakening interval one-way ANOVAs performed on each EEG parameter failed to distinguish any of the recall groups at the $p<.025$ level of significance. However, when evaluated at the $\mathrm{p}<.05$ level, there was a significant difference between groups on the EMG measure $E(3,62)=3.03, \mathfrak{p}=.036$. Post hoc comparisons of 
means using the Newman-Keuls procedure indicated lower amounts of EMG activity for the contentless group relative to the failure to recall group. The distibution of the means for the selected sample was in the same direction as the distribution of means when all cases were included. That is, the contentless group had the lowest amount of EMG activity followed closely by the success group, while the delayed success and failure groups were most similar and both showing the greatest amounts of EMG activity (See Table 2). In addition, results indicated a nonsignificant trend distinguishing groups on the alpha first derivative measure $F(3,62)=2.63, p=.058$. The contentless group had the lowest amount of alpha first derivative activity relative to the other groups.

For the postawakening interval, results of ANOVAs indicated a significant main effect of recall for the theta half-wave zero cross asymmetry measure $E(3,62)=3.12, \mathfrak{p}=.002$. Post hoc comparisons of means using the Newman-Keuls procedure revealed that the contentless group was associated with greater theta activity in the right hemisphere while failure to recall was associated with greater activity in the left hemisphere. In addition, the success group was significantly different from the failure group on that measure (See Table 2). However, the sample mean for the success group $(\underline{n}=18)$ did not approximate the mean recorded when 50 cases were used $(\underline{M}=-0.60(\underline{n}=50))$. Thus, this latter difference was considered unreliable. At the $p<.05$ level, results indicated a nonsignificant trend differentiating groups on the mean total power measure $(\underline{F}(3,63)=2.684, p=.054)$. The distribution of the means indicated 
the delayed group had the greatest amount of power in relation to the other groups (see Table 3). Separate ANOVAs on difference scores failed to distinguish any of the recall groups.

A closer examination of the distribution of the means for each group on the various EEG variables failed to reveal any consistent relationship between the four recall groups. For example, the success and failure groups were not always extremes, nor were the delayed and contentless groups always intermediate as hypothesized. Thus, there did not appear to be any systematic manner in which to combine the groups for further analysis.

2.4. Discussion and implications of findings

\subsubsection{Preawakening}

Proponents of models based on cortical activation (Antrobus, 1986; Hobson, 1988; Koukkou \& Lehmann, 1983; Pivik \& Foulkes, 1968) have generally attributed variations in dream recall to changes in EEG as they affect either the dream production, encoding (consolidation) or retrieval processes. Of the three, the production and encoding processes of the preawakening interval have been viewed as the most critical in determining recall performance. Although, Koukkou and L,ehmann (1983) described dream recall failure as a retrieval problem, that is, a difficulty experienced upon awakening, their model linked the inaccessibility of the dreamed experience to the quality of encoding during sleep. The emphasis placed on the preawakening EEG state to account for dream recall was due to the consistent and predictably higher rates of dream recall following awakenings from the corticaliy activated REM sleep 
Table 2. Means and standard deviations indicating significant* main effects of recall on electrophysiological (EEG) measures.

Recall categories

$\begin{array}{lcrrrr}\text { Preawakening_EEG } & & \text { Success } & \text { Contentless } & \text { Delayed Success } & \text { Failure } \\ \text { EMG } & \text { M } & 1932.00 & 1841.50 & 2263.11 & 2535.11 \\ & \text { SD } & 479.22 & 492.81 & 661.73 & 1062.98\end{array}$

Rostawakening EEG

Success Contentless Delayed Success Failure

$\begin{array}{llrrrr}L: R \text { theta } & \mathrm{M} & -3.72 & -3.17 & 0.00 & 2.22 \\ \text { zero cross } & \mathrm{SD} & 6.04 & 4.24 & 3.70 & 5.32\end{array}$

${ }^{* p}<.05$, two-tailed

Note: L:R represents interhemispheric ratio measure. The minus sign indicates greater activity in the right than in the left hemisphere: the plus sign indicates the opposite pattern of activity. The units of analysis for the EEG measures are arbitrary and arise from the algorithm used in the A/D converters. 
Table 3. Means and standard deviations for each recall group on postawakening electrophysiological (EEG) total power measure.

\begin{tabular}{lcccccc} 
& \multicolumn{5}{c}{ Recall categories } \\
\cline { 4 - 7 } Restawakening EEG & & Success & Contentless & Delayed Success & Failure \\
Total power & M & 729.00 & 694.15 & 909.56 & 709.22 \\
& SD & 256.11 & 229.52 & 318.31 & 188.55
\end{tabular}

Note. The units of analysis for the EEG measures are arbitrary and arise from the algorithm used in the $A / D$ converters. 
relative to other stages of sleep. However, in this study, the findings do not support the hypothesis of an increase in recall with corresponding increases in cortical activation recorded at parietal sites (see hypothesis 1a). For the preawakening interval, there were no significant group differences (success vs failure) in activation indexed by the EEG measures of power, and time spent in a given frequency band. However, in line with expectations there was a weak trend in which lower total power was associated with successful recall, while nonrecall tended to be related to higher total power. The relatively similar EEG state during the preawakening period would suggest a similar level of engagement of the production and/or encoding systems for both recall groups. Consequently, this finding suggests that as proposed by Koukkou and Lehmann (1983) dreaming may be equally ongoing during the preawakening interval for both recall groups. Moreover, it would appear that within a given sleep state the EEG activation level was not sufficient to account for differential dream recall performance.

The lack of differences in activation between recall groups may not rule out the use of cortical activation models in explaining variations in dream production and encoding during sleep, particularly evident between sleep stages. REM sleep is a more activated state than stages 2,3 or 4 . The main feature distinguishing reports following REM from other stages of sleep is that of length (Wollman \& Antrobus, 1987). Dreams remembered from NREM sleep are typically shorter than those from REM sleep. Furthermore, absence of dreaming, contentless reporting and single units dream 
fragments occur more frequently following NREM than REM awakenings (Foulkes, 1985). Generally, it appears that the main source of differences between stages is one of quantity of recall rather than quality (Foulkes, Bradley, Cavallero \& Hollifield, 1989). Antrobus (1986) and Foulkes (1985) have argued that due to the lesser amount of activation available in NREM sleep, the dream production and/or encoding processes are more limited relative to REM sleep and that this would account for the differential degree of reporting between stages. However, Wollman and Antrobus (1987) found no relationship between arousal and quantity of words recalled within a given sleep stage. Despite these findings, the level of cortical activation may play a role in allowing either a more continuous or extended production of dreams and/or of their encoding but cannot be taken as the sole determinant of their recallability. This conclusion is in agreement with observations that even within REM sleep, a state that appears to comprise the necessary and sufficient conditions to generate dreams, individuals can still experience a total lack of recall (Goodenough, 1978; Meir. 1989). In sum, dream recall should be considered a distinct process from dream generation and encoding processes.

In this study, there was also a trend for successful recall to be associated with lower muscle tone while nonrecall was related to higher muscle tone during the preawakening interval. Explanations to account for the relationship between dream recall and EMG variations are limited. The EMG measure is a sensitive index of muscle activation which is used to record both tonic discharges and 
phasic bursts of activity. The bulk of the research relating muscle tonus to dream recall has focused on phasic muscle discharges reflecting dreamed speech events (Shimizu \& Tsuyoshi, 1986) or large body movements (Dement \& Wolpert, 1958; Gardner \& Grossman, 1973) signalling shifts in scenarios or the end of a dream episode. These studies have demonstrated that abrupt physiological changes may be related to changes in dream recall. In this study, all EMG artifacts produced by large body movements were removed from analysis, with no such movements occurring during the 5 minute preawakening interval. Therefore, the submental EMG recordings made in this study may include bursts of activity without artifact due to isolated small muscle contraction or twitches but more generally represent tonic or sustained background muscle activity. Larson and Foulkes' (1969) is the only study which examined changes in tonic EMG activity in relation to dream recall in NREM sleep. Contrary to their findings, the present study suggested greater recall with lower muscle activity. Larson and Foulkes aimed specifically at investigating muscle inhibition just prior to REM sleep onset. Thus, their low EMG stage 2 intervals were sampled at a time of transition between states. Although the muscle activity was typically lower at those moments, the various changes in physiological activity accompanying the onset of REM sleep (Steriade \& McCarley, 1990) may have overridden any simple differences in muscle tonus in affecting recall. The finding of lower recall rates with lower EMG activity in Foulkes and Larson's (1969) study may have confounded the effects of the shift in state generally with those 
of muscle activity.

The presence or absence of submental muscle activity has also been examined in relation to speech during dreaming (Shimizu \& Tsuyoshi, 1986). In that study, the speech muscle activity recorded just prior to awakening corresponded to both short bursts of phasic discharges or continuous discharges. Shimizu and Tsuyoshi (1986) reported a higher incidence of speech in dreams in the presence of speech muscle phasic activity than in absence of such activity following both REM and stage 2 sleep awakenings. If a sufficient amount of phasic discharges was contained in our tonic EMG measure, it is possible that the amount of dreamed speech woald account for EMG differences between recall and nonrecall groups. Although some bursts of phasic discharges were contained in the tonic EMG recordings of this study, the contribution from this kind of activity was not substantial, nor is there evidence that it should be associated with recall performance.

An alternative explanation of differences in tonic muscle activity was provided by Cohen (1979) who suggested that general muscular tension during sleep may interfere with dream recall by reducing the salience of the dreamed experience as well as by introduring competing inputs during the process of recall. Cohen (1979) proposed that individuals who may be somewhat more "restless, twitchy of otherwise motorically engaged" (p. 176) may contribute on-line interference during the dreaming process which may in turn hamper dream recall. The findings of this study would lend support to such a notion. However, more research is needed to 
establish the effects of tonic EMG activity on dream recall, and contents of dreams.

At the preawakening interval, the differences in overall cortical activation reflected in the total power measure were minimal. The nonsignificant trend toward lower activation levels by the nonrecallers in comparison to recallers suggest there may be some differences between the two groups at the production or encoding levels but that these differences were clearly not sufficient to predict dream recall. In addition, the trend toward greater muscle activity for the nonrecallers than recallers implies that physiological systems other than EEG may affect (impede or facilitate) dream recall performance.

Yet, there is neurophysiological evidence (Tulving, Risberg, \& Ingvar, cited in Vaccarino, 1990) indicating that during wakefulness, the posterior region of the brain may be involved in semantic memory retrieval whereas the frontal areas may be related to episodic memory retrieval. Therefore, if such localizations of memory systems apply during sleep, then the lack of differences between recall groups at the parietal sites may suggest a similar involvement of the semantic memory retrieval system in both recall groups. Thus, EEG recordings made at other electrode placements, particularly the frontal areas may be needed to distinguish recall groups.

Semantic memory contains general information about logical relationships, rules and procedures, and facts about the world; that is, it corresponds to the organized verbal and non-verbal abstract 
knowledge (Baddeley, 1990; Kesner \& Di Mattia, 1987). Episodic memory contains information about the context in which an event has occurred in that it provides temporal and personal associations. Episodic memory is therefore self-referential or autobiographical (Baddeley, 1990). It plays an important role in the conscious experience of memory and is somewhat similar to working memory (Kesner \& Di Mattia, 1987). Episodic memory relates to events that have been completed and differs from working memory which deals with events in progress. During dreaning, the two memory systems (semantic and episodic) or two modes of processing may be required and equally activated to produce the dreamed experience. However, during dream recalling and reporting, there may be a bias toward the episodic/working memory modes of retrieval. Of the two, the episodic memory has been situated in the frontal lobes where information may be actively upgraded (working memory). Lesions to the frontal lobes would appear not to affect the encoding or storage of mnemonic material (Stuss \& Benson, 1986) but would impair memory processes including attention, motivation, programming and verification. These deficits in performance have beer. associated mainly to a heightened susceptibility to interference. That is, these deficits reflect an overall "inability to maintain consistent, directed attention over time based on an inability to control interfering stimuli" (Stuss \& Benson, 1986, p.192). In the context of dream recall for example, the prr ess of maintaining the necessary effort for recall, and of switching from one state to another (sleeping to waking) while retaining the dreamed experience may be 
impaired if the EEG activity of the frontal areas is somehow diminished. Thus, an examination of frontal sites may be necessary to assess differences in recall performance. Differences between recall groups would not be strictly one of production or of encoding but of transferring the mnemonic traces to wakefulness.

However, it may not be sufficient to examine activation levels at the frontal sites separately from other sites. A more adequate approach may be the examination of the relationship between the frontal and posterior regions of the brain. It may be possible to postulate two systems of memory which can operate independently of one another while the neural unis of analysis may be represented by their interconnections.

Recently, Nielsen, Petit, Montplaisir, Godbout \& Abel (1991) used intrahemispheric coherence measures to index int-rconnections between cortical areas. Coherence is an estimate of correlated activity, possibly of shared information between 'wo sites. It can be measured within specific frequency categories and is independent of signal amplitude (O'Connor \& Shaw, 1978). Nielsen et al. (1991) found high mean total EEG power spectrum coherence between the frontal and occipital sites and between the frontal and parietal sites, over the right and left hemispheres respectively. In addition, the two frontal comparisons showed increasing EEG coherence with a corresponding increase in sleep stages. Thus, REM had the least coherence followed by stage 2 , while stages 3 and 4 showed the most coherence. The higher coherence values for NREM sleep 'say be due to increasingly higher levels of low frequency EEG activity in those 


$$
2 \text { of/de } 2
$$


stages relative to REM sleep. However, the differential patterns of interconnectedness between frontal and posterior cortical areas across sleep stages may well be associated with differential recall rates for those sleep stages. Similarly, within a given stage, it may be that dream recallers depict different intrahemispheric coherence levels than nonrecallers. Thus, it may be worthwhile investigating the level of coherence between posterior and anterior sites in all frequency categories in relation to dream recall (Dummermuth \& Scollo-Lavizarri, 1972). Consistent with the functional role of the frontal areas in regulating the temporal ordering and sequencing of behaviors as well as maintaining working memory in attentional processes, a strong antero-posterior interconnection may be necessary to ensure that local processes activated posteriorly (vision, audition, somatosensory processing) be coordinated and integrated frontally. During sleep, poor dream recallers may show lower interconnectedness between anterior and posterior sites within a particular frequency band.

\subsubsection{Postawakening}

Very little information exists relating dream recall to postawakening EEG intervals. However, the postsleep EEG corresponds to the period closest to the retrieval of the sleep experience. As such it may reflect the carry-over effects of the preawakening state or constitute an entirely different processing state affecting the retrieval of the dreamed material.

For the postawakening interval, the findings do not support the hypothesis of greater recall with increasing activation, in terms of 
power measures (hypothesis $1 \mathrm{~b}$ ). The distribution of the means for total power did not show the expected decrease of activation (higher total power) with decreasing recall. In contradiction to the proposal of a carry-over effect mechanism, the success and failure to recall groups showed practically equal amounts of delta power. The distinctions in theta power followed the expected relationship with recall associated with less theta power relative to nonrecall; however, the differences were not large and failed to reach statistical significance. Awakenings made following the accumulation of slow wave sleep with its high amounts of low frequency activity are typically associated with confusion, disorientation and increased difficulty or failure to recall. This study failed to distinguish recall from nonrecall along any EEG measures of delta and theta activity. Therefore, the lack of recall may not be explained by a simple carryover effect of low frequency activity from the preawakening to the postawakening interval.

Upon awakening, there was significantly higher muscle tone and lower sigma first derivative activity in association with recall while nonrecall was related to lower muscle tone and higher sigma activity. The higher levels of EMG associated with recall were likely movement artifact due to the greater number of utterances provided by subjects who report their dreams and may not reflect a different level of alertness between recall groups. In the transition from wakefulness to sleep, increases in sigma activity, most apparent with stage? sleep in the form of spindles, characterize the onset of sleep. The reticular nucleus of the thalamus has been identified as the 
spindle generator and the main source of widespread inhibition of thalamocortical neurons during synchronized sleep (stages 2, 3 and 4) (Steriade \& McCarley, 1990). The reticular thalamic nucleus projects to virtually all thalamic nuclei as well as to more diffuse cortical connections. Spindling activity is transmitted through the thalamus over the cortex by thalamocortical neurons. Thus, the appearance of EEG spindle waves results in a powerful blockage of information transmitted through the thalamus (Steriade \& McCarley, 1990). This mechanism may allow a closing of sensory pathways (Steriade, 1983), a condition ensuring a genuine shift into sleep. Upon arousal, brainstem reticular stimulation quickly blocks spindling activity and transforms the oscillatory and inhibitory mode in thalamocortical circuits into a relay functional mode with tonically enhanced firing rates of cortical neurons. The cortical facilitation may be caused by an opening of synaptic transmission through thalamic relays. According to Steriade (1983), the blockage of spindle activity during the transition to arousal may involve a disruption by brainstem ascending inputs and basal forebrain axons to the reticular nucleus of the thalamus. This neuronal activity upon awakening presumably allows a greater receptivity to sensory information and readiness to respond. Thus, the results point to the greater alertness level of subjects who recall their dreams upon awakening relative to those who fail to recall.

Moffitt et al. (1982) examined differences between dream recallers and nonrecallers for a four minute postawakening interval. Their study indicated significant differences between recall groups 
(success vs failure) in alpha and beta frequencies but not the sigma frequency, for subjects who were asked to rehearse their sleep experience prior to reporting. Increases in alpha may be related to increases in intentive cognitive processing (focusing on internal processes) and those in beta may be associated with general information processing activity (Pigeau, 1983; Ray, 1984). Moffitt et al's findings may reflect a different cognitive style of recallers and nonrecallers recorded after an extended period of wakefulness. In the light of these findings, changes in sigma activity with recall in the 30 second postawakening interval, may be a precursor to changes in alpha and beta frequencies which may reflect greater waking activity. Overall, the results of this study would suggest that subjects who failed to recall their dreams manifested greater drowsiness upon awakening than those who were able to recall their dreams. This notion is in accord with findings of greater mean orientation time with nonrecall (time interval starting when the subject's name is called until the beginning of his report to the question "what was going through your mind"), relative to recall (Larson \& Foulkes, 1969; Pivik \& Foulkes, 1968).

Taken together, the findings of this study and those of Moffitt et al. (1982) suggest that postawakening, it is changes in the higher frequency categories, not slow wave activity that was significantly related to recall performance. Although low frequency activity may render dream recall more difficult (see delayed recall), the retrieval process may be more closely associated with the appearance of higher frequency activity, beginning with changes in the sigma band 
followed by changes in alpha and beta bands.

In sum, the finding of greater sigma EEG activity in association with nonrecall in comparison to recall provides evidence that dream recall may at least partially be a function of differential retrieval from memory in the postawakening period. Indeed, in the transition from sleep to wakefulness, sigma activity influences the transmission of information at thalamic and cortical sites. In addition, the difference in sigma first derivative activity between recall groups was recorded a short time after awakening. This suggests that the critical time interval influencing recall of dreams may be very short and close to the transition period between sleeping and waking.

At the postawakening interval, it was assumed that the arousal level at the time of awakening allowed consolidation started during sleep to progress even further (Goodenough, 1978). Similarly, time spent focusing on the dream experience or rehearsing the dreamed material would ensure the creation of more stable mnemonic patterns or more effective consolidation of the long term memory traces. An alternative explanation to the consolidation theory, not related to arousal, was that unless the dreamer was awakened during sleep to get a cue for retrieval from the short term memory stores, recall was said to be difficult or impossible. Therefore, due to the fragile nature of the short term memory trace, distractions upon awakening or conversely lack of focused attention would interfere with recall. Thus, it may be necessary to examine the relationship between EEG and attentional factors upon awakening. Activity in the sigma frequency band may be one such component affecting the 
ability to focus or to organize thoughts in the transition between sleeping and waking. Time spent awake has been found not to account for increased retention (Goodenough, Sapan, Cohen, Portnoff, \& Shapiro, 1971).

The limited number of significant EEG findings at the postawakening interval may indicate that the transition between sleep and wakefulness is a fragile state with a high susceptibility to interference and that other variables such as attention may play a role in determining dream recall. All in all, the postawakening interval appears to constitute a most critical period affecting recall performance.

\subsubsection{Preawakening to postawakening difference scores}

The difference scores computed in this study were used to index the magnitude of change in EEG from sleep to postsleep wakefulness. It was hypothesized that the ability to transfer information between dreaming and waking may be related to the electrophysiological similarity between the two EEG states (Koukkou \& Lehmann, 1983). However, the second hypothesis (hypothesis 2) stating that the greater the magnitude of EEG differences between the preawakening and postawakening intervals, the more impaired the recall, was not supported. The change scores characterizing the transition from sleep to wakefulness failed to differentiate the recall groups, except for a greater magnitude of change in sigma first derivative activity and muscle tone in association with recall than nonrecall. However, given the similar level of activation of the two groups during sleep, the changes in sigma and EMG activity were 
most likely due to differences registered at the postawakening interval. These findings cortradict Koukkou and Lehmann's view stating that subjects who recall their dreams should exhibit a state closer to wakefulness during sleep. Rather, upon being called, dream recallers appeared to move out of sleep and awaken more quickly.

In the present study, the changes between states were measured using differences between corresponding preawakening and postawakening scores within a given frequency band. Yet, it is known that the organization of neuronal activity of the brain consists of the interaction of patterns of EEG waveforms. Therefore, transfer of information across states may not be adequately reflected in terms of magnitude of change. The description of patterns of intercorrelations across frequencies within recall groups, such as presented by Moffitt and Hoffinann (1987) may provide a more appropriate descriptive means of examining differences between recall groups.

\subsubsection{Contentless and delayed success recall groups}

Overall, the hypothe:ses relating differences between success, contentless, delayed success and failure to recall groups were not supported at any of the three time intervals examined. There were no systematic trends for the means of the delayed success and contentless groups to show intermediate values of EEG activation between the success and failure groups. However, the small sample size used to investigate differences between groups may have precluded the emergence of significant findings. Therefore, only the general patterns of findings will be discussed. 
At the preawakening interval, there was a trend for the contentless group to show lower muscle tone and alpha first derivative activity than the failure to recall group. In addition, at the postawakening interval an asymmetry measure disinguished the contentless from the failure group at a significant level. However, the variability for all groups was large thus diminishing the strength of association of recall performance to a particular hemisphere. Nevertheless, at the postawakening interval, the balance of activity between hemispheres may well be involved in the experience of contentless reporting. Taken together, the contentless experiences as the most distinct, should not be combined with the failure reports but rather treated as a separate category of analysis. The contentless group had the smallest number of observations but its variability was comparable to the other groups. Thus, these findings may indicate some reliable trends that would need to be further examined.

At the postawakening interval, there was a :oonsignificant trend $(\mathfrak{R}<.054)$ indicating that the delayed group had a higher amount of total power than the other groups, due to its higher levels of lower frequency activity (alpha, theta and delta). Both the delayed success and contentless groups had equivalent amounts of total power at the preawakening interval but only the delayed success group retained high amounts upon awakening. These tentative trends suggest that at the postawakening interval, total power may affect some aspects of recall, primarily by prolonging the time needed to access the dreamed contents. Taken together, findings for the delayed and 
contentless recall suggest that dream recall performance may not be described as a simple linear progression from nonrecall to delayed, contentless and successful recall. More research is needed to clarify the association between various states of arousal upon awakening. EEG activity and dream recall.

\subsubsection{Asymmetries}

The hypothesis of no hemispheric asymmetry differences at the preawakening interval (hypothesis 3a) was supported. Thus, the theoretical proposition of Koukkou and Lehmann (1983) of greater left hemisphere involvement providing a facilitative effect upon recall was not supported. These findings are congruent with previous studies (Moffitt et al., 1982; Williamson et al., 1986) and suggest no differential involvement of the hemispheres in relation to dream recall at the production/encoding interval.

Although there were no differences noted between hemispheres, there is evidence indicating that intrahemispheric EEG activity should be examined in relation to dream recall. Thatcher, Krause \& Hrybyk (1986) obtained consistently greater coherence values within the right hemisphere than the left hemisphere. These authors explained that cortical connections of the left hemisphere may favour transfer of information within cortical regions while the right hemisphere may be more specialized for the processing of information across regions. Thus the right hemisphere consists of more long distance fibres to allow its long distance interactions while the left hemisphere with its more analytical and sequential processing requires a higher degree of short distance associations. 
Thus, it may be that a measure relating the activity between two electrode sites within the same hemisphere (i.e. antero-posterior sites) may help clarify the roles of each hemisphere in relation to the transfer of information in recalling dreams. That is, antero-posterior activity levels examined over the right and left hemisphere separately, within individual frequency bands, may reveal differential patterns associated with recall performance. This approach could be applied to both preawakening and postawakening EEG data sets.

The lack of differences between recall groups on any of the laterality measures at the postawakening interval failed to support the hypothesis of increasing left hemisphere involvement with increasing recall (see hypothesis $3 \mathrm{~b}$ ). The findings did not support the hypotheses outlined for the delayed and contentless recall groups (hypothesis 3c). However, contentless reporting may involve differential laterality between hemispheres. Generally, the lack of differences between groups suggests that broad distinctions such as recall/no recall may not be sufficient to distinguish a specific engagement of one or the other hemisphere.

For both the preawakening and postawakening intervals, it may not be the recall of dreams generally that is lateralized but rather some features of it. This notion was supported by Ehrlichman et al. (1985) and Moffitt et al. (1982). There may be a closer or different association of particular aspects of the report's content with laterality measures which remains to be investigated. 


\subsubsection{Gender}

There were no hypotheses formulated relating gender to recall performance given the exploratory nature of this variable. Overall there were few distinctions relating gender to dream recall. Both males and females showed a similar organization of EEG activity in relation to recalling dreams. At the preawakening interval, there was a trend towards greater theta power for female recallers than male recallers. Since there were no corresponding differences in the amount of time spent in the theta band, it is suggested that the power differences reflected the greater theta amplitude level in female recallers. The finding of greater amplitude in a low: frequency category for females in comparison to males is congruent with findings by Dijk et al. (1989). However, it is not clear at this time why greater theta amplitude would differentiate gender groups for successful recall only. Typically, theta activity during sleep is related to low activation states. During wakefulness, it has been viewed as reflecting attentional factors, particularly degrees of sustained attention in problem solving tasks. During sleep, as suggested by Pigeau (1985), high amplitude theta may be associated with a greater degree of vigilance albeit occurring at lower levels of activation. The latter proposal would be adequate but only for females. Although there were no statistically significant differences between recall and nonrecall, females showed a trend toward higher levels of theta power with successful recall than failure to recall, whereas males showed the opposite trend of lower levels of theta power with recall than nonrecall. More research is needed to clarify 
the relationship between gender, EEG activity in the theta frequency band and drearn recall.

The main effects for gender were recorded on the beta first derivative laterality measure and on the sigma first derivative measure. The latter is in agreement with findings by Gaillard and Blois, 1981, Dijk et al. (1989) and Flor-Henry et al. (1984) who reported a significantly greater density of spindle activity in the sleep of females than males throughout the night. It was suggested that the greater spindle density in females could be related to the slightly better stability of sleep in females generally.

The findings at the postawakening interval failed to distinguish males from females in terms of EEG activity averaged between hemispheres. Rather, the significant interaction between gender and recall performance was on the asymmetry measure of alpha power. On that measure, irrespective of whether they failed or succeeded in recalling a dream, the males showed a predominance in the left hemisphere, while the females depicted an opposite pattern of hemispheric predominance between recall and nonrecall. Given the lack of differences between groups in average amount of alpha activity, these findings of asymmetry may reflect gender differences in cognitive style. That is, males and females may differ in their tendency to use particular cognitive strategies (i.e. verbal/analytic left hemisphere or visual/synthetic right hemisphere with intentive/attentive modes of processing), when attempting to recall a dream (Pigeau, 1983; 1985). Based on these findings there is little evidence to suggest not combining males and females in a research 
study examining recall performance. However, information is lacking to clearly eliminate possible confounds due to gender. Therefore in future research it is still advisable to include a sufficient number of male and female subjects to allow gender cumparisons.

\subsection{Summary}

This research focused on the relationship between dream recall and EEG-defined cortical activation in an attempt to verify proposals outlined by Koukkou and Lehmann (1983) within the framework of their functional state-shift hypothesis. The bulk of research linking cortical activity to dream recall defined the preawakening EEG interval, thought to reflect the cortical activation level at the time of encoding of the sleep experience, as the primary condition determining the recall of dreams upon awakening. The present study does not support the hypothesis linking increased dream recall to increases in cortical activation prior to awakening. One implication of this finding is that the notion of a strict dependence of recall performance on the quality of EEG at the encoding period is untenable. However, the similarity of EEG states between recall groups supports Koukkou and Lehmann's (1983) proposal of equally ongoing dream generation and encoding processes for both groups. Consequently, dream recall and dream generation/encoding may be two independent processes with different neural mechanisms. The significant findings at the postsleep interval imply that accessibility to the dreamed material may primarily be a postawakening process. Indeed, this study indicated that one contributing factor in dream recall may be the opening of the thalamic gate upon awakening 
allowing for the transfer of information to the cortex. It was then suggested that high frequency activity may be differentially implicated as arousal and information processing progressed toward full wakefulness. The lack of findings in terms of magnitude of change between the preawakening and postawakening intervals does not entirely refute Koukkou and Lehmann's (1983) notion of similarity betwesn states as facilitating recall. In this study, the magnitude of change consisted of difference scores between corresponding sleep and postsleep EEG measures within a particular frequency category. It may be necessary to examine the interrelations of EEG variables across frequencies to fully describe the patterning of activity associated with the transfer of information between sleeping and waking states.

In sum, the present study revealed that dream recall from stage 2 sleep was not strongly determined by levels of cortical activation, as indexed by EEG measures at the preawakening period. For this encoding interval, briefer events are obscured when averaged over a five minute interval. It is possible that, immediately prior to awakening, shorter EEG events may more clearly distinguish recall from nonrecall. However, Williamson et al, (1986) examined 32 second EEG intervals prior to awakening and failed to differentiate recall groups. Therefore, it is unlikely that the examination of shorter time intervals will reveal group differences not detected using a longer period of time, at least up to 5 minutes. In addition, Meir (1989) found EEG differences between recall groups using a between subject analysis but failed to distinguish the groups 
following a within subject procedure. Thus, given a sufficient sample size, future research may profit from the use of within subject analyses to control for possible individual differences. Similarly, the examination of correlations between brain regions using both measures averaged over hemispheres and within the same hemisphere may be useful in characterizing the arousal-dream recall relationship. The study of dream recall should not be limited to the broad classification of reports into recall and nonrecall. Although tentative, there were trends suggesting arousal levels may vary in relation to the kinds of experience available upon awakening such as delayed recall and contentless reporting. All in all, sleep and dream research may benefit from the examination of changes in the pattern of arousal from the dreaming to the waking state. 


\section{References}

Antrobus, J. (1978). Dreaming for cognition. In A.M. Arkin, J.S. Antrobus, \& S. Ellman (Eds.), The mind in sleep. Hillsdale, NJ: Erlbaum.

Antrobus, J. (1983). REM and NREM sleep reports: Comparison of word frequencies by cognitive classes. Psychophysiology, 20, 562-568.

Antrobus, J. (1986). Dreaming: Cortical activation and perceptual thresholds. The Journal of Mind and Behavior, 7(2-3), 193211.

Antrobus, J. (1987). Cortical hemisphere asymmetry and sleep mentation. Psychological Review, 24(3), 359-368.

Antrobus, J.S., \& Ehrlichman, H. (1981). The "dream" report: Attention, memory, functional hemispheric asymmetry, and memory organization. In W. Fishbein (Ed.), Advances in sleep research: Vol.6. Sleep dreams and memory (pp. 135-145). New York: Spectrum Publications.

Antrobus, J., Ehrlichman, H., Wiener, M., \& Wollman, M. (1983). The REM report and the EEG: Cognitive processes associated with cerebral hemispheres. In W.P. Koella (Ed.), Sleep 1982. Sixth European Congress on Sleep Research. Basel: S. Krager.

Armitage, R. (1986). Ultradian rhythms in EEG al. performance: An assessment of individual differences in the basic rest-activity cycle. Unpublished doctoral dissertation, Carleton University, Ottawa.

Armitage, R., Hoffmann, R., Loewy, D., \& Moffitt, A. (1989).

Variations in period-analyzed EEG asymmetry during REM and NREM sleep. Psychophysiology, 26, 329-336. 
Aserinsky, E., \& Kleitman, N. (1953). Regularly occurring periods of eye motility, and concomitant phenomena, during sleep. Science, 118, 273-274.

Baddeley, A. (1990). Human memory: Theory and practice. Hove and London: Lawrence Erlbaum Associates.

Baekeland, F. (1970). Correlates of home dream recall. The Journal of Nervous and Mental Disease, 150(3), 209-214.

Bakan, P. (1977). REM sleep and the right hemisphere: A theoretical integration. Journal of Altered States of consciousness, 3 , 285-307.

Benjamin, L. (1963). Statistical treatment of the law of initial values (LIV) in autonomic research: A review and recommendation. Psychosomatic Medicine, 25, 556-566.

Bertini, M., Violani, C., Zoccolotti, P., Altomare, P., Dorrichi, F., \& Evangalisti, L. (1985). Lateralization of verbal and configurational processing during waking and upon awakenings from REM and NREM. In W. Koella, E. Ruther \& $\mathrm{H}$. Schulz (Eds.), Sleep '84 (pp. 358-360). New York: Gustav Fischer Verlag.

Boisacq-Schepens, N. (1983). Sensory perception. In M. Monnier \& M. Meulders (Eds.), Functions of the nervous system; Vol.4. Psycho-neurobiology (pp. 449-484). Amsterdam: Elsevier.

Broughton, R. (1975). Biorhythmic variations in consciousness and psychological functions. Canadian Psychological Review, 16, 212-239.

Bryden, M.P. (1982). Laterality: Functional asymme $f y$ in the intact brain. New York: Academic Press. 
Church, M., March, J., Hibi, S., Benson, K., Cavness, C., \& Feinberg, K. (1975). Changes in frequency and amplitude of delta activity during sleep. Electroencephalography and Clinical Neurophysiology, 39, 1-7.

Cohen, D. (1972). Failure to recall dream content: Contentless vs dreamless reports. Perceptual and Motor Skills, 34, 10001002.

Cohen, D. (1974). Toward a theory of dream recall. Psychological Bulletin, 81, 138-154.

Cohen, D. (1979). Sleep and dreaming: Origins, nature and functions. Oxford: Pergamon Press.

Cohen, J., \& Cohen, P. (1983). Applied multiple regression/ correlation analysis for the behavioral sciences. Hillsdale, NJ: Lawrence Erlbaum Associates.

Cronbach, L., \& Furby, L. (1970). How we should measure "change:or should we? Psychological Bulletin, 77, 68-80.

Darling, M. (1989). The pattern of self-reflectiveness in dream reports. Unpublished master's thesis, Carleton University, Ottawa.

Dement, W., \& Kleitman, N. (1957a). Cyclic variations in EEG during sleep and their relation to eye movements, body motility and dreaming. Electroencephalography and Clinical Neurophysiology, 2, 673-690.

Dement, W., \& Kleitman, N. (1957b). The relation of eye movements during sleep to dream activity: An objective method for the study of dreaming. Jcurnal of Experimental Psychology, 53, 339-346.

Dement, W. \& Wolpert, E. (1958). The relation of eye movements, body motility, and external stimuli to dream content. Lournal of Experimental Psychology, 55, 543-553. 
Dijk, D., Beersma, D., \& Bloem, G. (1989). Sex differences in the sleep EEG of young adults: Visual scoring and spectral analysis. Sleep, $12(6), 500-507$.

Doricchi, F., Violani, C., \& Milana, I. (1990). Hemispheric lateralization of cognitive functions in high frequency and low frequency dream recallers. Sleep Research, 19, 143.

Dummermuth, G., \& Scollo-Lavizzari, G. (1972). Spectral analyses of sleep EEG in normal adults. In W.P. Koella \& P. Levin (Eds.), Sleep: Physiology. Biochemistry. Psychology. Pharmacology. Clinical implications. First European Congress on Sleep Research. Basel: S. Karger.

Eccles, J.C. (1977). The understanding of the brain. New York: McGraw-Hill.

Ehrlichman, H., Antrobus, J.S., \& Wiener, M.S. (1985). EEG asymmetry and sleep mentation during REM and NREM mentation. Brain and Cognition, 4, 477-485.

Farah, M., Gazzaniga, S., Holtzman, I., \& Kosslyn, S. (1985). A left hemisphere basis for visual imaging? Neuropsychologia, 23, 115-118.

Fein, G., Floyd, T.C., \& Feinberg, I. (1981). Computer measures of sleep EEG reliably sort visual stage 2 epochs by NREM period of origin. Psychophysiology, 8(6), 686-693.

Fenigstein, A., Scheier, M.F., \& Buss, A. (1975). Public and private self-consciousness: Assessment and theory. Journal of Consulting and Clinical Psychology, 43, 522-527.

Fiss, H. (1969). Current dream research: A psychobiological perspective. In B.B. Wolman (Ed.), Handbook of dreams: Research. theories and applications. New York: Van Nostrand Reinhold Company. 
Fiss, H., Klein, G., \& Bokert, E. (1966). Waking fantasies following interruption of two types of sleep. Archives of General Psychiatry, 14, 543-551.

Flor-Henry, P., Koles, Z., \& Reddon, J. (1984). Age and sex related EEG configurations in normal. In A. Glass (Ed.), Individual differences in hemispheric specialization (pp. 121-148). New York: Plenum Press.

Foulkes, D. (1962). Dream reports from different stages of sleep. Journal of Abnormal and Social Psychology, 65(1), 14-25.

Foulkes, D. (1966). The psychology of sleep. New York: Charles Scribner's \& Sons.

Foulkes, D. (1978). A grammar of dreams. New York: Basic Books.

Foulkes, D. (1985). Dreaming: A cognitive-psychological analysis. Hillsdale, NJ: Lawrence Erlbaum Associates.

Foulkes, D., Bradley, L., Cavallero, C., \& Hollifield, M. (1989). Processing of memories and knowledge in REM and NREM dreams. Perceptual and Motor Skills, 68, 365-366.

Foulkes, D. \& Fleischer, S. (1975). Mental activity in relaxed wakefulness. Journal of Abnormal Psychology, 84, 66-75.

Foulkes, D., \& Vogel, G. (1965). Mental activity at sleep onset. Journal of Abnormal Psychology, 70, 231-243.

Gabel, S. (1988). The right hemisphere in imagery, hypnosis, rapid eye movement sleep and dreaming. The Journal of Mental Disease, 176, 323-331.

Gaillard, J-M., \& Blois, R. (1981). Spindle density in sleep of normal subjects. Sleep, $4(4), 385-391$.

Galaburda, A, LeMay, M., Kemper, T. \& Geswind, N. (1978). RightLeft asymmetries in the brain. Science, 199, 852-856. 
Galin, D. (1974). Implications for psychiatry of left and right cerebral specialization. Archives of General Psychiatry, 31, 572-583.

Gardner, R., \& Grossman, W. (1973). Normal motor patterns in sleep in man. In E. Weitzman (Ed.), Advances in Sleep Research, Vol 2. (pp.67-107). New York: Spectrum Publications.

Goodenough, D.R. (1978). Dream recall: History and current status of the fieid. In A.M. Arkin, J.S. Antrobus \& S.J. Ellman (Eds.), The mind in sleep (pp. 113-140). Hillsdale, NJ: Lawrence Erlbaum Associates.

Goodenough, D.R., Lewis, H.B., Shapiro, A., Jaret, L., \& Sleser, I. (1965). Dream reporting following abrupt and gradual awakenings for different types of sleep. Journal of Personality and Social Psychology, 2, 170-179.

Goodenough, D, Sapan, J., Cohen, H., Portnoff, G., \& Shapiro, A. (1971). Some experiments concerning the effects of sleep on memory. Psychophysiology, $\underline{8}(6), 749-762$.

Goodenough, D.R., Shapiro, A., Holden, M., \& Steinschriber, L. (1959). Eye movements, electroencephalograms, and the recall of dreams. Journal of Abnormal and Social Psychology, 58-59, 295-302.

Greenberg, M.S., \& Farah, M.J. (1986). The laterality of dreaming. Brain and Cognition, 5, 307-321.

Greenwood, P., Wilson, D., \& Gazzaniga, M. (1977). Dream report folowing commissurotomy. Cortex, 13, 311-316.

Hall, C. (1984). "A ubiquitous sex difference in dreams" revisited. Journal of Personality and Social Psychology, 46(5), 109-117.

Hartmann, E. (1984). The Nightmare: The psychology and biology of terrifying dreams. New York: Basic Books. 
Herman, J.H., Ellman, S.J., \& Roffwarg H.P. (1978). The problem of NREM dream recall re-examined. In A.M. Arkin, J.S. Antrobus \& S.J. Ellman (Eds.), The mind in sleep (pp. 59-92). Hillsdale, NJ: Lawrence Erlbaum Associates.

Herman, J., Rampy, P., Hirshkowitz, M., Tharpe, G., Bliss, K., \& Roffwarg, H. (1987). EEG asymmetry during REM sleep, waking imagery, and mentation. Sleep Research, 17, 232.

Herman, J., Rampy, H., Roffwarg, H., Homan, R., \& Hirshkowitz, M. (1989). Asymmetric EEG activity in temporal and parietal cortex during REM sleep in humans. Sleep Research, 18, 22.

Hobson, J.A. (1988). The dreaming brain. New York: Basic Books.

Hobson, J.A., Lydic, R., \& Boghdyan, H.A. (1986). Evolving concepts of sleep cycle generation: From brain centers to neuronal populations. The Behavioral and Brain Sciences, 2, 371-448.

Hobson, J.A., \& McCarley, R.W. (1977). The brain as a dream state generator: An activation-synthesis hypothesis of the dream process. American Journal of Psychiatry, 134, 1335-1348.

Hoffmann, R., Moffitt, A., Shearer, J., Sussman, P., \& Wells, R. (1979). Conceptual and methodological considerations towards the development of computer-controlled research in the electrophysiology of sleep. Waking and Sleeping, 3, 1-16.

Hoffmann, R., Moffitt, A., Wells, R., Sussman, P., Pigeau, R., \& Shearer, J. (1984). Quantitative description of sleep stage electrophysiology using digital period analytic techniques. Sleep, 1, 356-364.

Kamiya, J. (1961). Behavioral, subjective, and physiological aspects of drowsiness and sleep. In D.W. Fiske \& S. R. Maddi (Eds.), Functions of varied experience (pp. 145-174). Homewood, Illinois: The Dorsey Press. 
Kesner, R., \& Di Mattia, B. (1987). Neurobiology of an attribute model of memory. In A. Epstein \& A. Morrison (Eds.), Progress in psychobiology and physiological psychology (pp. 207-277). London: Academic Press.

Klatzky, R. (1984). Memory and awareness: An informationprocessing perspective. New York: W.H. Freeman \& Co.

Kolb, B., \& Winshaw, I. (1980). Eundamentals of human neuropsychology. San Francisco: W.H. Freeman and Co.

Koukkou, M., \& Lehmann, D. (1968). EEG and memory storage in sleep experiments with humans. Electroencephalography and Clinical Neurophysiology, 25, 455-462.

Koukkou, M., \& Lehmann, D. (1980). Brain functional states: Determinants, constraints and implications. In $\mathrm{M}$. Koukkou, K. Lehmann \& J. Angst (Eds.), Functional states of the brain: Their determinants (pp. 13-20). Amsterdam: Elsevier/NorthHolland Biomedical Press.

Koukkou, M., \& Lehmann, D. (1983). Dreaming: The functional stateshift hypothesis. British Journal of Psychiatry, 142, 221-231.

Koukkou, M., \& Lehmann, D. (in press). A model of dreaming and its functional significance: The state-shift hypothesis. In $A$. Moffitt, M. Kramer, \& R. Hoffmann (Eds.), The functions of dreaming. Albany: State University of New York Press.

Koulack, K., \& Goodenough, D.R. (1976). Dream recall and dream recall failure: An arousal-retrieval model. Psychological Bulletin, 83(5), 975-984.

Larson, J.D., \& Foulkes, D. (1969). Electromyogram suppression during sleep, dream recall, and orientation time. Psychophysiology, 5(5), 548-555. 
Laughlin, C., McManus, J., Rubinstein, R., \& Shearer, J. (1986). The ritual transformation of experience. Studies in Symbolic Interactions, 1 (Part A), 107-136.

Lehmann, D. (1980). Fluctuations of functional state: EEG patterns and perceptual and cognitive strategies. In M. Koukkou, D. Lehmann \& J. Angst (Eds.), Functional states of the brain: Their determinants (pp. 189-202). Amsterdam: Elsevier/North-Holland Biomedical Press.

Lehmann, K., Dummermuth, G., Lange, B., \& Meier, C.A. (1981). Dream recall related to EEG spectral power during REM periods. Sleep Research, 10, 151.

Lehmann, D., \& Koukkou, M. (1984). Physiological and mental processes during sleep: A model of dreaming. In $\mathbf{M}$. Bosinelli \& P. Cicogna (Eds.), Psychology of dreaming. Bologna, Italy: CUUEB.

Linn, R., \& Slinde, J. (1977). The determination of the significance of change between pre- and posttesting periods. Review of Educational Research, 47, 121-150.

Lord, L. (1963). Elementary models for measuring change. In C. Harris (Ed.), Problems of measuring change. Madison: University of Wisconsin Press.

Matousek, M., \& Petersen, I. (1973). Frequency analysis of the EEG in normal children and adolescents. In P. Kellaway \& I. Petersen (Eds.), Automation of clinical electroencephalography. New York: Raven Press.

McCarley, R.W. (1981). Mind-body isomorphism and the study of dreams. In W. Fishbein (Ed.), Advances in sleep research: Vol.6. Sleep. dreams and memory (pp.205-237). New York: Spectrum Publications. 
McCarley, R.W. \& Hobson, J.A. (1975). Neuronal excitability modulation over the sleep cycle: a structural and mathematical model. Science, 189, 58-60.

Meier, B. (1989). Psychophysiological correlates of REM dream recall. Sleep Research, 18,149.

Moffitt, A. \& Hoffmann, R. (1987). On the single-mindedness and isolation of dream psychophysiology. In J. Gackenbach (Ed.), Sleep and dreams: A sourcebook. New York: Garland.

Moffitt, A., Hoffmann, R., Wells, R., Armitage, R., Pigeau, R., \& Shearer,

- J. (1982). Individual differences among pre- and postawakening EEG correlates of dream reports following arousals from different stages of sleep. The Psychiatric Journal of the University of Ottawa, Z(2), 111-125.

Murri, L., Mancino, M., Massetani, R., Canapicchi, R., Puglioli, M. \& Rossi, G. (1989). Effect of acute and chronic brain damage on dreaming. Research Communications in Psychology. Psychiatry and Behavior, 14(2), 121-142.

Niedermeyer, E. \& da Silva, L. (1982). Electroencephalography: Basic principles, clinical applications and related fields. Baltimore: Urban \& Schwerzenberg.

Nielsen, T., Petit, D., Montplaisir, J., Godbout, R., \& Abel, A. (1991) . Intrahemispheric EEG coherence during sleep and wakefulness: Interactions with gender and hand preference. Manuscript submitted for publication.

O'Connor, K., \& Shaw, J. (1978). Field dependence, laterality and the EEG. Biological Psychology, 6, 93-109.

Overton, D.A. (1978). Major theories of state dependent learning. In B.T. Ho, D.W. Richards, III, D.L. Chute (Eds.), Drug discrimination and state dependent learning, New York: Academic Press. 
Pigeau, R. (1983). A review of hemispheric EEG asymmetry and a proposed theory for the psychological and physiological correlates of EEG. Unpublished Ph.D. Comprehensive, Psychology Dept., Carleton University, Ottawa, 1983.

Pigeau, R. (1985). Psychophysiology of cognition: Some EEG correlates and a new descriptive technique. Unpublished doctoral dissertation, Carleton University, Ottawa.

Pigeau, R.A., Hoffmann, R.F., \& Moffitt, A.R. (1981). A multivariate comparison between two EEG analysis techniques: Periodanalysis and fast fourier transform. Electroencephalography and Clinical Neurophysiology, 52, 656-658.

Pivik, R.T. (1978). Tonic states and phasic events in relation to sleep mentation. In A.M. Arkin, J.S. Antrobus, \& S.J. Ellman (Eds.), The mind in sleep. Hillsdale, NJ: Lawrence Erlbaum Associates.

Pivik, R.T, Bylsma, B.A, Busby, K., Saivyer, M.A. (1982). Interhemispheric EEG changes: Relationship to sleep and dreams in gifted adolescents. The Psychiatric Journal of the University of Ottawa, Z(2), 56-76.

Pivik, T., \& Foulkes, D. (1968). NREM mentation: Relation to personality, orientation time, and time of night. Journal of Consulting and Clinical Psychology, 32(2), 144-151.

Pivik, T., Halper, C., \& Dement, W. (1969). Phasic events and mentation during sleep . Psychophysiology, 6, 215.

Purcell, S. (1987). The education of attention to dreaming in High and Low Frequency dream recallers. Unpublished doctoral dissertation, Carleton University, Ottawa.

Purcell, S., Mullington, J., Moffitt, A., Hoffmani, R., \& Pigeau, R. (1986). Dream self-reflectiveness as a learned cognitive skill. Sleep, 2(3), 423-437. 
Ray, W. (1984). Attentional factors and individual differences reflected in the EEG. In A. Glass (Ed.), Individual differences in hemispheric specialization (pp. 149-168). New York: Plenum Press.

Rechtschaffen, A. (1973). The psychophysiology of mental activity during sleep. In F.J. McGuigan \& R.A. Schoonover (Eds.), The psychophysiology of thinking. New York: Academic Press.

Rechtschaffen, A., \& Kales, A. (1968). A manual of standardized terminology, techniques and scoring system for sleep stages of human subjects. (National Institute of Health Publication No. 204) Washington, D.C.: United States Government Printing Office.

Rechtschaffen, A, Verdone, P., \& Wheaton, J. (1963). Reports of mental activity during sleep. Canadian Psychiatric Association Journal, $\underline{8}(6), 409-414$.

Reinsel, R., Antrobus, J., \& Fein, G. (1989). Hemisphere differences and sums in sleep and waking: Effects of state of consciousness, time of night, and electrode placement. Sleep Research, 18,21.

Rogosa, D., \& Willett, J. (1983). Demonstrating the reliability of the difference score in the measurement of change. Journal of Educational Measurement, 20(4), 335-343.

Rosa, D., Antrobus, J., \& Reinsel, R. (1989). Investigating the left hemisphere and right hemisphere EEG differences in sleep. Sleep Research, 18, 22.

Schachter, H. (1987). Psychophysiological relations during sleep: The role of individual differences. Unpublished doctoral dissertation, Carleton University, Ottawa.

Schanfald, D., Perli ıan, C., \& Greenberg, R. (1987). Focal brain damage and dream recall. Sleep Research, 14, 116. 
Schwartz, D.G., Weinstein, L.N., \& Arkin, A.M. (1978). Qualitative aspects of sleep mentation. In A.M. Arkin, J.S. Antrobus \& S.J. Ellman (Eds.), The mind in sleep (pp. 143-244). Hillsdale; NJ: Lawrence Erlbaum Associates.

Sharma, K., \& Gupta, J. (1986). Optimum reliability of gain scores. Journal of Experimental Education, 54, 105-108.

Shimizu, A., \& Tsuyoshi, I. (1986). Dreamed speech and speech muscle activity. Psychophysiology, 23, 210-214.

Simons, I., Ogilvie, R., Segalowitz, S., \& Janicki, M. (1990) EEG power spectrum changes during behaviorally indicated arousals from stage 2 sleep. Proceedings of the 4th Annual Meeting of the Association of Professional Sleep Societies (p 62). Minneapolis, MN.

Slap, J.W. (1977). On dreaming at sleep onset. The Psychoanalytic Quarterly, 46, 71-81.

Snyder, F. (1971). The psysiology of dreaming. Behavioral Science, 16, 31-44.

Spear, N.E., \& Gordon, W.C. (1981). Sleep, dreaming, and the retrieval of memories. In W. Fishbein (Ed.), Advances in sleep research: Sleep. dreams and memory (pp. 183-203). New York: Spectrum Publications.

Springer, S., \& Deutsch, G. (1989). Left brain, right brain. New York: W.H. Freeman and Co.

Steriade, M. (1983). Cellular mechanisms of wakefulness and slow wave sleep. In A. Mayes (Ed.), Sleep mechanisms and functions (pp. 161-216). Cambridge, Great Britain: Van Nostrand Reinhold.

Steriade, M., \& McCarley, R. (1990). Brainstem control of wakefulness and sleep. New York: Plenum Press. 
Stuss, D., \& Benson, D. (1986). The frontal lobes. New York: Raven Press.

Tabachnick, B., \& Fiddell, L. (1989). Using multivariate statistics. New York: Harper \& Row Publishers.

Thatcher, R., Krause, P., \& Hrybyk, M. (1986). Cortico-cortical associations and EEG coherence: A two-compartmental model. Electroencephalography and Clinical Neurophysiology, 64 , 123-143.

Tracy, R.I., \& Tracy, L.N. (1974). Reports of mental activity from stages 2 and 4. Perceptual and Motor Skills, 38, 647-648.

Vaccarino, F. (1990). Physiological psychology. In J. Grusec, R. Lockart \& G. Walters (Eds.), Foundations of psychology (pp. 40-82). Toronto: Copp Clark Pitman Ltd.

Verdone, P. (1965). Temporal reference of manifest dream content. Perceptual and Motor Skills, 20, 1253-1268.

Vogel, G.W. (1978). Sleep-onset mentation. In A.M. Arkin, J.S. Antrobus, \& S.J. Ellman (Eds.), The mind in sleep. Hillsdale, NJ: Lawrence Erlbaum Associates.

Weingartner, H. (1978). Human state dependent learning. In B.T. Ho, D.W. Richards III, D.L. Chute (Eds.). Drug discrimination and state dependent learning. New York: Academic Press.

Weisz, R. (1972). Phenomenological correlates of discrete events in NREM sleep: The K-complex as a NREM phasic indicator. Psychophysiology, 2, 127.

Williamson, P.C, Csima, A., Galin, H., \& Mamelak, M. (1986). Spectral EEG correlates of dream recall. Biological Psychiatry, 21, 717723. 
Williams, R. \& Zimmerman, D. (1977). The reliability of difference scores when errors are correlated. Educational and Psychological Measurement, 37. 879-689.

Wollman, M.C., \& Antrobus, J.S. (1987). Cortical arousal and mentation in sleeping and waking subjects. Brain and Cognition, 6, 334-346.

Wolpert, E.A. (1972). Two classes of factors affecting dream recall. Journal of the American Psychoanalytic Association, 20, 45-58.

Wolpert, E.A., \& Trosman, H. (1958). Studies in psychophysiology of dreams. Archives of Neurology and Psychiatry, 79, 603-606.

Zimmerman, W. (1970). Sleep mentation and auditory awakening thresholds. Psychophysiology, 6(5), 540-549.

Zimmerman, D., \& Williams, R. (1982). Gain scores in research can be highly reliable. Journal of Educational Measurement, 19 , 149-154.

Zimmerman, D., Williams, R., \& Zumbo, B. (in press). Effect of nonindependence of sample observations on parametric and nonparametric statistical tests. Psychological Bulletin. 


\section{Appendix A}

\section{Research Application Form}

The Sleep and Chronopsychology Lab is looking for volunteer Psychology 100 students to participate in a research project. The Lab is looking for right-handed males and females. All subjects will be asked to sleep one night in the lab and will receive four course credit points for their participation. If you already have some of your credits, then participating in this study will allow you to complete your credits. Volunteers must be free of any major sleep problems (e.g. any trouble getting to sleep at night or any trouble staying asleep tinrough the night), and must sleep between 7 and 8 hours regularly per night. Your participation will be greatly appreciated. If interested please enter the necessary information on the sign-up sheet provided. Thanks. 
Appendix B

\section{Follow-up Information}

In order to take part in this study, I need to know: Typically, how often do you remember your dreams upon awakening in the morning?

rarely, less than once a month...................
infrequently, about once a month...............
sometimes, one to two times per week....
often, two to five times per week...............
every morning with rare exceptions.......... Are you right- or left-handed? You will sleep one night in the lab. You will be told what to expect when you first come to the lab. You will be awakened a number of times during the night and asked to describe your sleep experience. Approximately, how many hours do you sleep at night? Are you having any trouble getting to sleep at night? Are you having any trouble staying asleep through the night? Do you have any major health problems? Are you currently taking any medication? Have you ever had your EEG recorded? I'll ask you to refrain from alcohol, caffeine and drug consumption on the day of the study. Also, I would like you not to take any naps unless you typically do so. Are you interested? If so, let's schedule you. You are encouraged to bring your own pillow, and may bring anything else you are comfortable with (covers, etc.). If not we are fully equipped. 
Appendix C

\section{Instructions Upon Arrival At The Lab}

During the night, you will be awakened a number of times and asked to describe anything that was happening before you heard me call your name. In this study, I am equally interested in whether you recall something or not. It is important that you report whatever you can recall just before awakening even if you do not recall a full dream, that is if there were just sounds or images or body sensations or even nothing at all. Describe whatever was going through your mind before I called your name. When you hear me calling your name, let me know that you are awake by saying 'yes I am awake' or 'OK I hear you'. After you give your report you will be asked to respond to a few questions. Let's discuss these questions now so that you will know what to expect during the night. If you have any other questions about what is going to happen, feel free to ask me now before we set you up for the night. You are free to withdraw from the study anytime you want to. 
Appendix D

\section{Consent Form}

\section{LABORATORY FOR THE STUDY OF SLEEP AND CHRONOPSYCHOLOGY}

Description of procedures:

Volunteers in this project are asked to spend one night sleeping in the laboratory chimber. For female volunteers, a female researcher will be present for the duration of the session.

Before the volunteer goes to bed, the researcher will attach electrophysiological electrodes on the scalp and facial skin of the volunteer using tape and collodion.

During the night, the researcher will monitor the volunteer's brain activity, muscle tension and eye movements from the instrument room. The volunteer will be able to speak with the researcher by voice intercom during the night. $A$ number of awakenings will be performed during the night in order to elicit a description of the sleep experience, which will be audio-taped. The volunteer will not be significantly deprived of sleep time.

Agreement to participate:

I, have read

(volunteer's name)

the above description and I agree to permit the researcher to carry out the required actions. I agree to hold them blameless for any inconveniences which my volunteering may cause me: eg. minor disruption of my normal sleep routines, etc. I agree that any data collected by the researcher during my time in the Sleep Laboratory may be used for scientific research and I understand that such data will not be publicly identified as my own. Confidentiality will be maintained to the greatest possible extent.

Date:

Signature:

Witness: 
Appendix E

\section{Wakeup Protocol}

1. [Name], wake up, keep your eyes closed and remain as still as you possibly can.

(Proceed once the subject shows clear sign of awakening: eg., a response, some movement, EEG changes, etc.).

2. Could you please describe with as much detail as possible, any experiences you were having before I called your name. Anything else?

When there is little or no recall, the subject will be asked to "think for a moment or so and see if anything comes back to you" (10-15 secs.).

Whether there is recall or no recall, proceed with probes 3 to 7 . 3. Where 1 is 'deep asleep' and 7 is 'wide awake', rate how you felt before you heard me call your name.

4. Where 1 is 'restless and agitated' and 7 is 'relaxed and sound', rate your sleep.

5. Where 1 is 'uninterested' and 7 is 'extremely interested', rate your motivation to recall any experience this wake-up.

6. Where 1 is 'easy' and 7 is 'difficult', rate the ease or difficulty you had in (trying to recall) recalling your experience.

7. What made your attempt to recall this experience 'easy/difficult'.

8. Thank you. You can go back to sleep now. 


\section{Appendix $\mathbf{F}$ \\ The self-consciousness scale}

Subject ID

Please rate each of the following items on a scale from zero to four, where zero indicates that the item is extremely uncharacteristic of you and four indicates that the item is extremely characteristic of you. Please be sure to answer all items by circling the appropriate number.

1. I'm always trying to figure myself out.

2. I'm concerned about my style of doing things.

3. Generally, I'm not very aware of myself.

4. It takes me time to overcome my shyness in new situations

5. I reflect about myself a lot.

6. I'm concerned about the way I present myself.

7. I'm often the subject of iny own fantasies.

8. I have trouble warking when someone is watching me.

9. I never scrutinize myself.

10. I get embarrassed very easily.

11. I'm self-conscious about the way I look.

12. I don't find it hard to talk to strangers. 
13. I'm generally attentive to my inner feelings.

14. I usually worry about making a good impression.

15. I'm constantly examining my motives.

16. I feel anxious when I speak in front of
a group.

$\begin{array}{lllll}0 & 1 & 2 & 3 & 4\end{array}$

17. One of the last things I do before I leave

- my house is look in the mirror.

$\begin{array}{lllll}0 & 1 & 2 & 3 & 4\end{array}$

18. I sometimes have the feeling that I'm off somewhere watching myself.

$\begin{array}{lllll}0 & 1 & 2 & 3 & 4\end{array}$

19. I'm concerned about what other people think of me.

$\begin{array}{lllll}0 & 1 & 2 & 3 & 4\end{array}$

20. I'm alert to changes in my mord.

$\begin{array}{lllll}0 & 1 & 2 & 3 & 4\end{array}$

21. I'm usually aware of my appearance.

$\begin{array}{lllll}0 & 1 & 2 & 3 & 4\end{array}$

22. I'm aware of the way my mind works when

I work through a problem.

$\begin{array}{lllll}0 & 1 & 2 & 3 & 4\end{array}$

23. Large groups make me nervous.

$\begin{array}{lllll}0 & 1 & 2 & 3 & 4\end{array}$ 


\section{Appendix $\mathbf{G}$ \\ Instructions to Raters}

Upon awakening subjects were asked: Could you please describe with as much detail as possible any experiences you were having before I called your name. If they appeared to have some recall of content subjects were repeatedly asked if they had anything else to report until they had nothing left to report. When subjects reported little (that they were not sure if any preawakening experience occurred or that they knew something occurred but they couldn't describe what it was or if they reported no recall), they were then asked to "think for a moment or so and see if anything comes back to you". The reports for each subject corresponds to a separate page. For each subject, the wakeups are presented in order and separated by an asterisk $\left({ }^{*}\right)$. The reports are to be classified into four categories:

1) Success: recall of some content attributed by subjects to the preawakening period. Reports could be of dreaming, thinking or feeling. The content may be a visual image, an experience in another sensory modality (with or without visual imagery) or an emotional experience (affect). Experiences describing thoughts related to the process of sleep are considered contentful. Ex: I felt I wasn't really asleep and thinking how I should be asleep. That would be a success. A success is coded 1.

2) Contentless: In answer to instructions subjects first claimed to have been dreaming or that something was going through their mind 
before being called but they couldn't say or describe what it was. No content was available there was an inability to recollect any details of the sleep experience. When asked to think about it for a moment subjects were still unable to recall anything at all.

A contentless is coded 2.

3) Delayed recall: This category is divided into delayed success and delayed contentless.

3.1) Delayed success: subjects first claimed nothing was happening or at least that they were not sure. When asked to think about it, subjects reported some content (see success).

A delayed success is coded 3.

3.2) Delayed contentless: subjects first claimed nothing was happening or at least that they were not sure. When asked to think about it, subjects claimed with a relative degree of certainty that something was happening but they couldn't say or describe what it was (see contentless). Reports of subjects who when asked to think about it, were uncertain and claimed that maybe there was something or said "yes I suppose there was something", are categorized as failures.

A delayed contentless is coded 5 .

4) Failure: after being given the opportunity to think about it, subjects were still unable to report anything at all. They didn't think anything was happening; their mind was blank; there was no impression of dreaming.

A failure is coded 4. 
